\title{
Studien zu den Weihgeschenken und der Topographie von Delphi. IV.
}

Von H. Pomtow.

Mit Beiträgen von H. Bulle.

Die Epigonen (Nr. 16).

1. Beschreibung des Halbrunds.

Das Epigonenhalbrund haben wir wegen zu groker Zerstörung nicht in den einzelnen Steinen und Quadern vermessen können; nur einige Haupt-

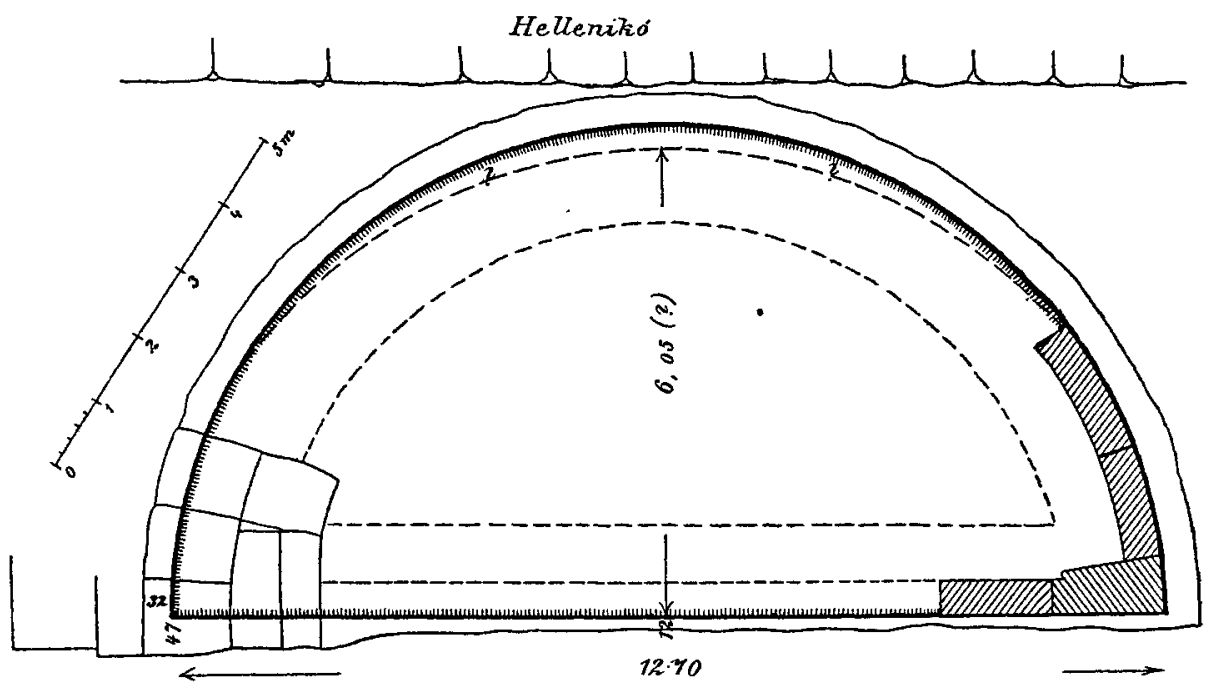

Abb. 9. Schematischer Grundriß des Epigonen-Halbrunds. - Maßsstab 1125.

make wurden genommen. Trotzdem sich mit Hilfe der Photographien eine hinreichende Vorstellung von dem heutigen Zustand der Ruine geben läßrt, ist der Wiederaufbau des Ganzen überaus schwierig, sowohl wegen der fehlenden Detail-Matie als auch besonders, weil von den Standplatten oder Aufschriften der Statuen nicht der geringste Rest vorhanden ist. Daher können für die Rekonstruktion hier nur allgemeine Hinweise gegeben werden, die zeigen, wie sich die vorhandenen Ueberbleibsel vielleicht verwerten lassen würden, wenn einst ein Architekt die Vermessung und den Wiederaufbau in die Hand nimmt. 
Eine Vorstellung des Erhaltenen gibt Abb. 1 auf Taf. III, die hoch oben von dem Nordrand der gegenüberliegenden Königsnische aufgenommen ist, sowie die schematischen Darstellungen von Grundrifs (Abb. 9) und Querschnitt (Abb. 10), die jedoch in den Maßen sehr verbesserungsbedürftig sind. Die freigegrabene Osthälfte des Unterbaus veranschaulicht Abb. 2 auf Taf. III, die erhaltene West-Ecke des Halbrunds Abb. 10 auf Taf. IV.

Der Fundamentbau. Bei den auf Abb. 2 sichtbaren, grob gekrönelten Steinlagen (aus Parnaßsstein) kann man zunächst Zweifel hegen, ob sie in situ sind oder etwa modern wieder aufgebaut, und einer unserer erfahrensten Olympia-Architekten glaubte zuerst beim Anblick der Photographie an modernen Wiederaufbau. Augenscheinlich ist aber der ganze Bau nach Süden zu (links auf Abb. 2) durch Erdbeben stark deformiert und eingesunken und das moderne Abgraben des stätzenden Erdreichs hat dann ein übriges getan, um die Fundamentlagen aus Lot und Flucht zu bringen. Rechts (an der Ost-Ecke) ist die Erhaltung eine bessere und hier erkennt man deutlich, dafs von den Quaderlagen des Unterbaus die 5 obersten in situ sind; mehr waren es auch im Altertum nicht, denn unter der fünften erscheint antiker Boden. Diese Fundamentlagen haben die Ausgrabenden beim Ausheben des Grabens unterfangen, indem sie regellos kleinere Steine und fremde Quadern (darunter keilförmige wichtige Stücke des Innen-Baus) unterstopften; daher wissen wir nicht, ob die zwei untersten Lagen (die 4. und 5.) ehemals da aufhörten, wo sie jetzt zu Ende sind, über der keilförmigen Platte links vorn, oder ob sie ebenso wie die 3 ober-

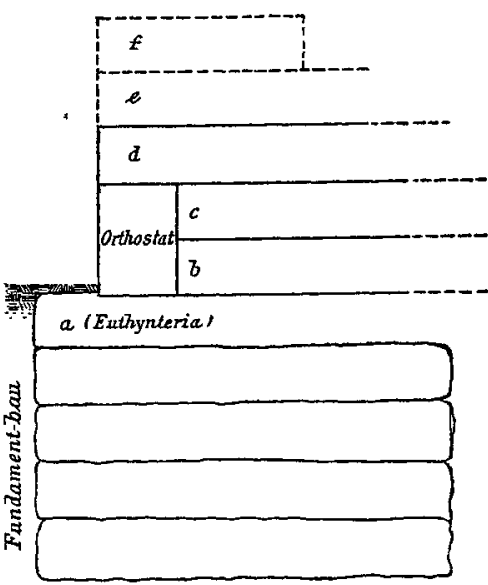
Abb. 10. Schematischer Schnitt durch die Peripherie des Halbrunds. $(1: 50)$. sten links unter dem ganzen Bau weiter

liefen. An sich wäre ersteres möglich, da die Fundamentierung im Osten höher sein mußte, als im Westen, aber da sie in unserem Fall nicht sichtbar und auch nicht stärker belastet war, erscheint dieser Grund nicht zwingend ${ }^{1}$ ).

Die hohl liegenden wagerechten Quadern ganz rechts in der Höhe der drei oberen Fundamentschichten gehören nicht zu den Epigonen, sondern sind die Reste des aus mehreren Lagen bestehenden, nicht fundamentierten Pavimentes des anstossenden Amphiaraos-Wagens, die soeben beschrieben wurden. Die Mauerwand ganz links ist die Innenseite des Hellenikó, dessen Quadern hier, wie auch Tafel I (Marathon) zeigte, nur roh bearbeitet worden sind; man sieht, dac der Bogen unseres Halbrundes im Süden (links) gegen sie gestoßen ist, daß also der ganze Bau absichtlich so berechnet war, daßis er den Raum zwischen Straße und Hellenikó

1) Auf Abb. 1 (Taf. III) sind in der Mitte noch 4 Quaderlagen unter den Orthostaten sichtbar, die Fundamentschichten scheinen dort also besser erhalten als aufen. 
genau fïllte. Ganz vorn (rechts) hat man wieder herrenlose Quadern in der Tiefe der Grabensohle zusammengestapelt, darunter Breitplatten von der Balustrade der Königsnische (vgl. Klio VII p. 400).

Dieser Fundamentbau war, wie Abb. 1 erkennen läst, nicht durchgeschichtet; er ist nur ein ringsum laufender Mantel, der einen Kern aus Erde umschlok. Seine Dicke betrug zwei Plattentiefen, d. h. etwa $1,80 \mathrm{~m} . \mathrm{Ob}$ die Fundamentschicht unter der Vorderkante längs der Strake ebenso stark ist, steht nicht fest, doch ist es aus verschiedenen Umständen wahrscheinlich. Daß der Unterbau auch im Westen vorhanden ist, ist selbstverständlich und auf Abb. 11 (Taf. IV) erkennbar; doch wird er hier vielleicht nicht so tief hinabreichen, wie im Osten.

Der Oberbau ${ }^{1}$ ) und das anfgehende Mauerwerk (Abb. 10). Obwohl die oberste Fundamentlage, die Euthynteria $(a)$ seitlich nicht die Abarbeitungslinie zeigt, die sonst die antike Niveauhöhe anzugeben pflegt (Athen. Mitt. 1906, 452, Mïnchener Sitzungsber. 1907, 258), ist sie doch als Ausgleichsschicht dadurch kenntlich, daß sie lïngs der Aubenrundung einen 0,32 breiten Rand enthält, der eine schwache, $2-3 \mathrm{~cm}$ tiefe Einbettungstäche ringsum einfabt. Sie trug an ihrer Peripherie die Orthostaten des aufgehenden Mauerwerks und ist auf den Abbildungen gut sichtbar. Diese Orthostaten $(b+c)$ bestehen aus 0,58 hohen, c. $0,40 \mathrm{~m}$ breiten schwach gerundeten Blöcken von wechselnder, z. T. gewaltiger Länge (bis zu 1,80), die an der Aussenseite glatt und für Ansicht berechnet waren, innen grob gekrönelt sind und oben glatten Saumschlag zeigen (Abb. 11). Ueberihnen folgte lïngs der Kreisperipherie keine zweite Orthostatenreihe, sondern eine c. 0,38 hohe Lagerschicht $(d)$ keilförmig geschnittener Platten, von denen noch eine in situ ist (die westlichste, Abb. 11); sie sind nach innen zu viel breiter (tiefer) als die Dicke der Orthostate, zeigen anscheinend oben gleichfalls Saumschlag und rauhen Spiegel, dienten also als Auflager für andere, jetzt verlorene Platten.

Inne $\mathrm{nb}$ a u und Paviment. Die oberste Fundamentschicht $(a)$ (Euthynteria) setzte sich wohl nach innen zu als Planum fort. Von dieser Fortsetzung sind Reste des zweiten inneren Plattenkranzes (links von der Mitte, Abb. 1) erhalten, an den Vorderecken vielleicht auch solche des dritten, nach innen zu folgenden. Ueber dieser Fläche lagerten längs der Peripherie noch zwei Plattenschichten $(b$ u. $c$ ) übereinander, deren

1) Wie viel von diesem Oberbau moderne Wiederherstellung ist, läßt sich nicht genau bestimmen; wahrscheinlich sind alle Orthostaten (außer an der Westecke) und die meisten über der Euthynteria lagernden Platten erst nachträglich wieder aufgebaut, die Westecke immer ausgenommen. Homolle sagt ausdrücklich von den 'Septem und Epigonen': ${ }_{n}$ l'ex-voto des Argiens, retrouvé et en partie recons tit ué sur la droite [vielmehr: gauche] de la route" (Bull. 21, 296), und daß bei dieser Rekonstitution leider auch viele Stücke der Königsnische fälschlich h i e r verbaut wurden, obwohl ihr Durchmesser gröfer ist, ist mehrfach bemerkt worden (Orthostaten und Breitplatten der Königsbalustrade, die z." T. noch jetzt im Epigonenhalbrund stehen und es verunzieren). Auch Bulle bestätigt mir, daf zur Zeit seiner Anwesenheit (1898 und 1903) k e in Stein des aufgehenden Mauerwerks mehr in situ war (aufer an der WestEcke), daß also alle diese Stücke modern wieder aufgebaut seien. 


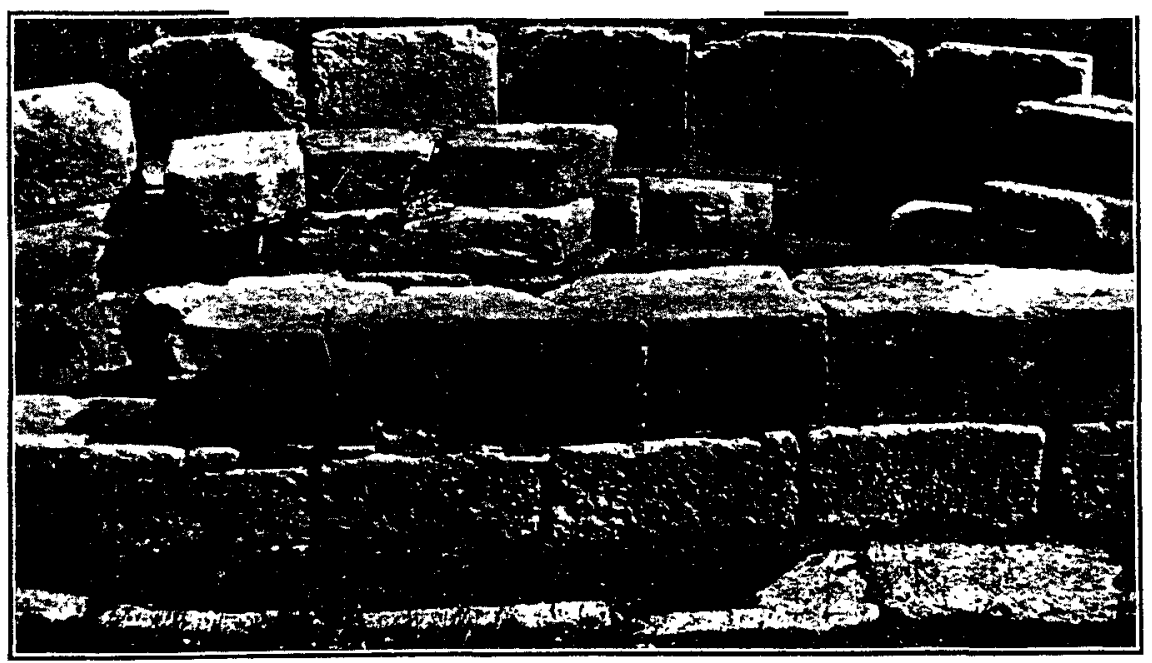

Abb. 5 .

Weihe-Insch

der

Sieben gegen $T 1$

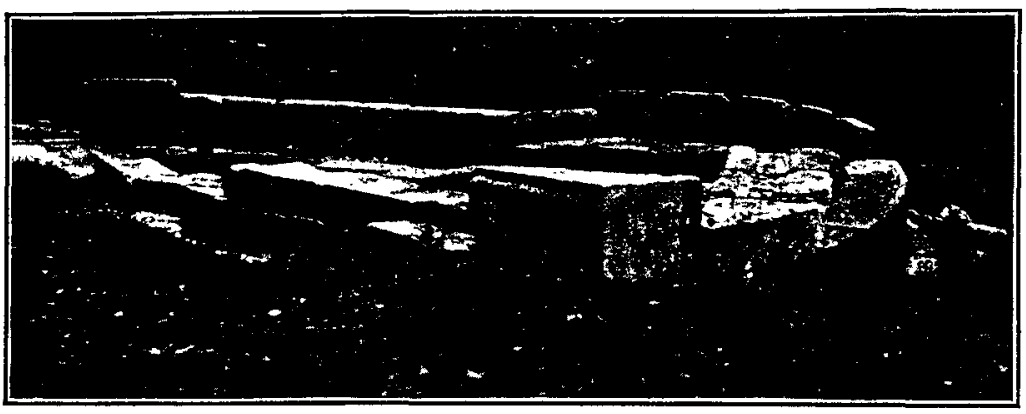

Abb. II.

Westecke

des

Epigonen-Halbrun
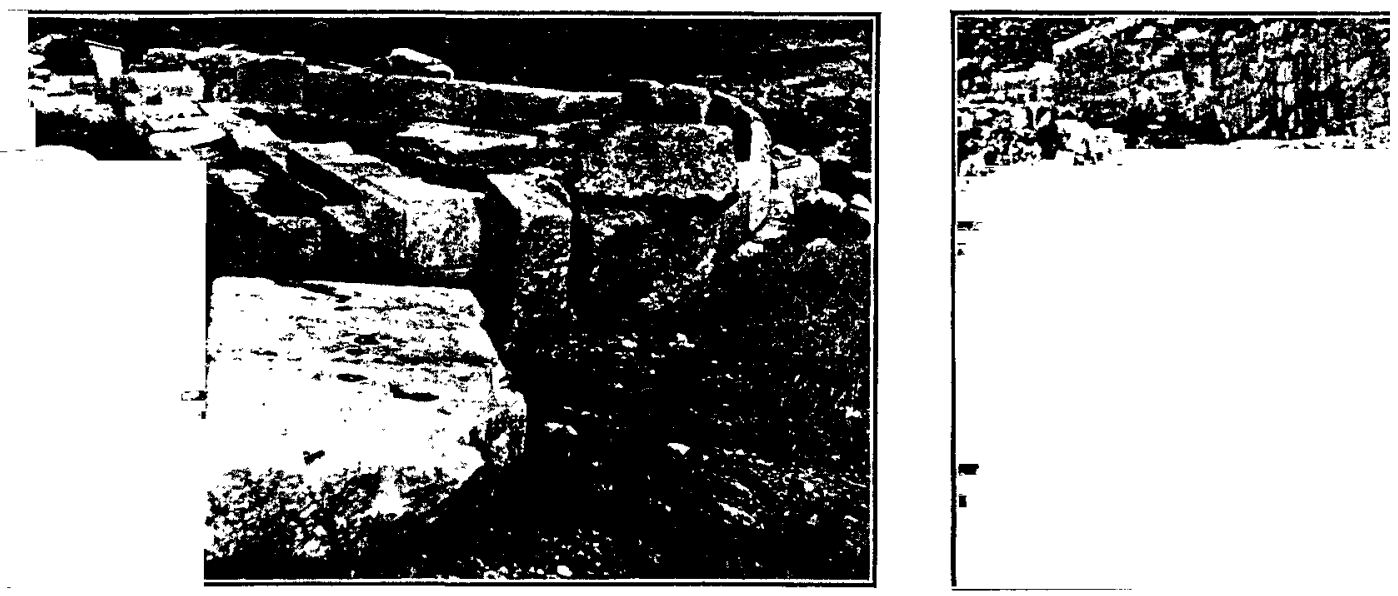

Abb. I2. Das Halbrund von Westen.

Abb. I3. Ecke der Tarentiner

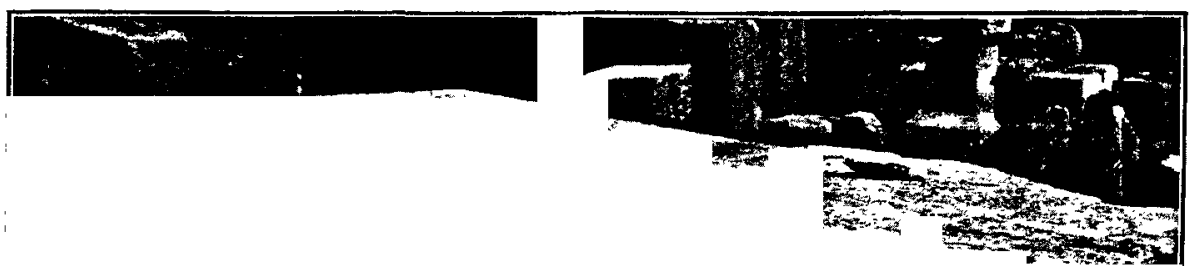

Abb.

Weihe - I

de

$\delta \varepsilon x a ́ \tau \alpha \nu$ 
Brought to you by | Université Paris Ouest Nanterre La Défense

Authenticated

Download Date | 4/2/17 11:16 PM 
Höhe zusammen genau die der Orthostaten erreichte; gegen diese sind sie gestoßen und schneiden mit deren Oberkante ab. Drei Platten der Schicht $b$ sind in der Mitte des Kreisbogens erhalten, vgl. Abb. 1, vor den 2 fälschlich hier aufgerichteten Balustradeplatten der Königsnische; eine größere Anzahl füllt das ganze westliche Drittel. Von der auf $b$ liegenden Schicht $(c)$ sind die Platten der West-Ecke vorhanden, auf sie greift die vorher genannte, über den Orthostaten folgende Lagerschicht (d) nach innen zu über, bezw. ruht auf ihr und den Orthostaten.

Welche von diesen 4 übereinander lagernden Schichten ( $a$ : Euthynteria, $b$ u. $c$ gegen die Orthostaten gestoben, $d$ über letzteren ruhend) als eigentliches Paviment (Trittfäche) gedient hat, ist nicht ohne weiteres sicher. Jedenfalls lagerten die Pavimentplatten nicht auf Quermauern oder Rosten. wie bei der Königsnische, da von solchen anscheinend nichts vorhanden ist, sondern der Innenkern wurde wahrscheinlich wie bei der Lysanderkammer aus Erde und groben großen Steinbrocken gebildet, deren Oberseite man platt abgeschlichtet hatte, um auf sie die Pflasterschicht zu legen. $\mathrm{Da}$ das Pflaster aber bei so einfacher Verlagerung nicht genügend fest (fugendicht) liegt, so wird man nach dem Beispiel der lyysanderkammer wenigstens zwei Plattenschichten übereinander anzunehmen haben, die untereinander verdübelt gewesen sind. Sie entsprächen den mit $a$ und $b$ bezeichneten. Noch eine dritte durchlaufende Pflasterschicht (c) darüber anzunehmen, wird man sich nur schwer entschließ3en, weil sie den Innenkern zu sehr belastet hätte, trotzdem scheint sie durch einige Indizien des vorderen Abschlusses gefordert zu werden.

Vorderer A bschluk. An der Vorderkannte längs der Straße ist das Denkmal fast ganz zerstört. Man erkennt auf Abb. 1, dał die linke Ecke ein gut Teil höher liegt, als die Mittelstrecke und die Westecke, und es ist schwer zu entscheiden, ob erstere durch Erdbeben gehoben ist, oder letztere gesunken sind ${ }^{1}$ ). Denn dab die Ostecke, an der nur die Platten der Euthynteria erhalten sind, nicht modern aufgebaut ist, was man zur Lösung der Schwierigkeit meinen möchte, beweist der Umstand, dafs diese Platten schon vor 20 Jahren genau ebenso schief lagen, als ich sie untersuchte ${ }^{2}$ ); sie bildeten damals das Pflaster des Hofes von Haus Nr. 127 (jetzt Nr. 525, Kap. Bottiglias), dessen Erbauung an der Zerstörung unseres Denkmals große Schuld hat, weil seine hohe Außentreppe nebst ihrem Podest und ein Teil seiner Fundamentmauern aus unsern gut behauenen, bequem gelegenen und dicht an dēr Erdoberfläche befindlichen Plattenlagen erbaut worden waren (vgl. Beiträge z. Topogr. v. D. p. 56). Es lag dicht östlich von unserm Halbrund da, wo die Septem gestanden haben müssen, und sein Kellergeschoß hat auch deren Reste z. T. zerstört. - Von dem

1) Die Senkung der Mitte scheint mir sicher. Sonst könnten die beiden Ecken nicht mehr so gut im Verband liegen. Bulle.

2) Ich liek damals Sondierungslöcher führen, um den Innenbau zu erkennen, fand aber zu unserm Erstaunen nirgends Platten, Fundamente oder Mauern, sondern überall nur Erde, auch nördlich des vorderen Abschlusses. Heut ist die Ursache des Mißerfolges klar, der ganze Innenkern war von jeher nur aufgefüllte Erde. 
vorderen Abschluf des Halbrunds ist wenigstens so viel sicher, dafi die Euthynteria in der ganzen Länge vorhanden ist. Die Breite ihres erhabenen Außenrandes wechselt hier so stark, daß sie links an der Ecke 0,47, in der Mitte nur 0,12 beträgt und in letzterer Breite bis zum Westende durchläuft. Was hent auf der Euthynteria liegt (Abb. 1), scheint modern hingelegt; sicher ist das der Fall bei der viel herumtransportierten Inschriftstufe (mit den quadratischen Löchern), die oben (Abschn. 5) beschrieben war, und bei den östlichsten Quadern der unter ihr lagernden Schicht, zweifelhaft bei deren Mittelstrecke, die rechts mit der von hinten bis vorn durchgehenden, über der Euthynteria ruhenden Schicht $b$ bündig zu sein scheint. Ueber die vorn rechts erscheinenden Orthostaten ist bei der Beschreibung der Westecke zu handeln.

Die Westecke des Halbrunds. Abb. 11 und 12 (Taf. IV) geben die Westecke wieder, die von dem ganzen Bau am besten erhalten ist und allein Winke für die Rekonstruktion zu geben vermag ${ }^{1}$ ). Wir erkennen, dak die vordere Abschlufwand hier aus denselben Orthostaten besteht, wie das übrige Halbrund, dał aber über ihnen vorn auf der Ecke eine zweite Orthostatenschicht $(=d+e)$ steht, deren Eckstein in situ ist. $\mathrm{Er}$ ist vorn grade, hinten schräg (keilförmig) geschnitten, rechts gerundet, dem Ansatz des Halbkreises entsprechend, links anscheinend rechtwinklig eingearbeitet. Die Vorderseite zeigt sehr schöne, gleichmäßige Krönelung und einen rings herum laufenden vertieften sauberen Randschlag (den Lehrstreifen), rechts und links je eine flache Bosse. Alles zeigt an, dak diese Seite für Ansicht berechnet war. Dagegen scheint der darunter stehende, gleichfalls mit Hebebossen versehene Orthostat $(=b+c)$, der etwas länger ist und weiter nach Osten reicht (Abb. 1), ehemals wie heute z. T. im Erdreich gesteckt zu haben. Seine Fortsetzung nach Osten zu ist in einer, vielleicht modern hierhergestellten Orthostatquader erhalten, die ebenso, wie der oberste Eckstein, auf der Oberseite Auflagerfläche hat. Letzterer ist anscheinend ein wenig höher als die unteren Orthostaten, etwa $0,60-0,65$ hoch [?].

In der Mitte der Vorderseite hat man auf einer niedrigen Stufe, die von dem Einbettungsrand der Euthynteria um etwas mehr als die Orthostatendicke nach Süden zurückgenommen ist, die großen Quadern der Argiver-Inschrift verlagert. Da sie aber, wie sich am Sehluti der Septem herausgestellt hat, gar nicht zu den Epigonen gehört, so haben wir uns um diesen modernen Wiederaufbau hier nicht weiter zu kümmern, sondern unabhängig von ihm den Wiederherstellungsversuch zu unternehmen.

2. Wiederherstellungsversuch.

Aus der Art der Fundamentierung ergibt sich deutlich, daß der Zentrumsteil des Halbrunds entlastet und leer war, der breite und schwere Aukienkranz aber nicht nur zum Stützen des inneren Erdreichs diente, sondern selbst zum Tragen bestimmt war. Denn in seiner ganzen Breite

1) Die auf Abb. 5 (Taf. IV) rechts vor der Westecke sich erhebenden Blöcke sind die Eckpolygone der Tarentinerterrasse, die Platten im Vordergrund die $\delta \varepsilon x \dot{x} \tau \alpha \nu$-Steine (s. u.). 
lastet ein niedriger Oberbau auf ihm, dessen untere beiden Lagen $(b$ u. $c$ ) von den runden Orthostaten wie von einem steinernen Reifen umfalt und in ihrer Lage unverrückbar festgehalten werden. Es ist nun mehrfach von mir daranf hingewiesen worden, daf die Anlage von Kammern und Nischen lediglich aus technischen und topographischen Gründen geschah - um Raum am Berghang auszusparen - nicht aus künstlerischen. In ganz Delphi ist nicht ein einziges Beispiel bekannt, das ein freistehendes Anathem von einem $\mathrm{k}$ ü $\mathrm{s}$ s $\mathrm{l}$ i ch e $\mathrm{n}$ Hintergrund umgeben zeigte. Hinterwände, Kammern, Nischen etc. finden sich $\mathrm{n} u \mathbf{r}$ an den Nordseiten der Wege und Plätze, nach der Bergsteigung zu, ni emals an den Südseiten; vgl. Daochos-Monument, Alexanderjagd, Kammer bei Thor 3 etc. Darum kann kein Zweifel sein, daf über dem Fundamentbau der Epigonen keinerlei Umfassungswände emporgingen, sondern dak das Ganze nur dazu erbaut war, um den Sockel eines Statuen-Halbkreises zu tragen und dessen Basen gegen jede Verschiebung durch Erdbeben oder dergl. zu sichern. Für die Wirkung des Ganzen war es unerläßlich, daßs die Bildsäulen etwas über das Strafenniveau emporgehoben wurden, und hierfür waren zwei bis drei weitere Sockelstufen nötig, von denen eine erhalten $(d)$, die zweite erschlossen ist $[e]$. Da der Statuensockel an den beiden Enden natürlich nicht ganz bis zur Strabe reichte, so erhielten die vorderen Ecken einen anders gearteten, schönen und kräftigen Abschluß in Gestalt von Anten, die aus 2-3 Orthostaten übereinander bestanden.

Zwischen diesen Anten mufs der Zugang zum Halbrund gelegen haben. Da auch die ganze Vorderseite fundamentiert war - wenigstens ist die oberste Fundamentlage (a) durchgängig erhalten -, also gleichfalls zum Tragen gedient hat, andrerseits aber kein Sockelbau hier gestanden haben kann, so ergibt sich mit Notwendigkeit die Annahme einer, wenn auch ganz niedrigen Vorderwand, die von Ante zu Ante reichte und von der die gerade Orthostatplatte (rechts) den einzigen erhaltenen Rest bildet. Da sie oben Auflager zeigt, folgte noch eine Schicht (d) über ihr. Nach Analogie der Lysanderkammer, der Argosnische etc. wird Lage $d$ simsartig über den Orthostat vorgekragt haben (etwa $10 \mathrm{~cm}$ ) und man darf sie mit großer Wahrscheinlichkeit als das eigentliche Paviment betrachten. Denn es ist kein zwingender Grund vorhanden, über $d$ noch weitere, durchlaufende Quaderschichten anzunehmen; vielmehr spricht die Wahrscheinlichkeit dafür, daß man die Steinpackungen des Oberbaues möglichst gering machte, um den Unterbau zu entlasten.

An sich wäre es möglich, daß́ die lose Orthostatplatte noch zur Ante selbst gehörte, diese also eine Breite von 2 Quadern gehabt habe, und dafi zwischen den so verbreiterten Anten eine Freitreppe emporführte, in der ganzen Breite der Vorderwand. Wir haben uns mit einer derartigen Rekonstruktion so lange abgequält, als sie durch das Vorhandensein der Inschriftstufen nahegelegt und bedingt wurde. Indessen blieb 'die Anbringung der Inschrift an der Treppe, statt am Statuensockel doch höchst auffällig und ohne Analogie. Denn die Inschriften an den Stufen der Athenerhalle und des Siphnierschatzhauses sind $k$ e in e Analoga, weil dort die ganzen Ge bä u d e geweiht werden, hier aber die Statuen' (Bulle). Seit aber die 
Inschriftstufen ausgeschieden sind, treten die allgemeinen Erwägungen wieder in ihr Recht und sie lassen eine Verbreiterung der Anten und auch die Annahme einer Freitreppe als beispiellos und fremdartig ablehnen.

Man wird nach Analogie der gegenüberliegenden Nischen für wahrscheinlich halten, dafs der Eingang zu unserm Halbrund im Osten lag, dafs also dicht neben der Ost-Ante eine kleine zweistufige, höchstens $1 \mathrm{~m}$ breite Treppe der Vorderwand vorgelagert oder in sie eingeschnitten war, wie wir ersteres bei der Lysanderkammer, letzteres bei der Argosnische angenommen haben (Klio VII, $401 \mathrm{f}$., $444 \mathrm{f}$.). Im übrigen ist es keineswegs ausgemacht, dafs die 4 Lagen $a-d$ ganz durchgeschichtet waren. Man würde nach dem Beispiel des daneben liegenden Pflasters des AmphiaraosWagens 2-3 durchgehende Pflasterlagen für mehr als genügend halten. Vielleicht war die Euthynteria $(a)$ nicht durchgeschichtet, sondern bildete nur ebensolchen Plattenkranz, wie die Lagen des Unterbaues. Ja, es braucht sogar keine einzige der Schichten $a-d$ a priori als durchgehendes Pflaster aufgefakt zu werden und zur Unterstützung dieser Ansicht könnte man auf Furtwänglers Worte hinweisen: 'auch die Epigonen standen auf einem offenen halbkreisförmigen Posta me nt' (Mïnch. Sitzungsber. 1901, 402). Indessen war dies gesagt im Gegensatz zu der Aufstellung in Kammern und Nischen und schliebt nicht mit Notwendigkeit die Vorstellung eines Erd-Fußbodens innerhalb unseres Halbrunds ein. So werden wir in Rücksicht auf die Stärke unseres Unterbaues und auf die Analogie des zeitlich am nächsten stehenden Lysander-Paviments v o rl ä ufig 2-3 Pflasterlagen übereinander voraussetzen, ohne der späteren Beispiele der halbrunden $\mathrm{kl}$ e in en Exedren (auch Nische Nr. 11, Klio VII, 435 f.) oder der Argoskammer als Analoga zu bedürfen.

Ueber den Statu e n s o ckel läßrt sich vermuten, daßs er nicht wie bei der Argosnische nur aus einer, 0,30 hohen Stufe bestand, unter der eine breitere ganz flache Unterstufe $(0,12)$ hervorsah, sondern daß die Statuen, weil sie sich gegen den Himmel projizierten, ein wenig höher standen als vor einer Hintergrundwand. Daher möchte ich glauben, dak die zu ergänzende Lage $e$, die ehemals mit dem erhaltenen obersten Orthostat der West-Ante in gleicher Höhe abschlof, die untere ziemlich hohe Bathronstufe $(0,35)$ war. Die nächste Lage $(f)$ über ihr wurde wohl schon durch die Standplatten der Statuen gebildet und treppte gegen $e$ vorn $a b$, wie bei der Königsnische. Dal noch ein schmaler Au $\hat{B}$ enring von niedrigen Abschlußsteinen sie einfakte oder etwas überragte, wird man gern annehmen wollen, doch hat sich anscheinend kein Rest von ihm erhalten ${ }^{1}$ ). Dieser abschließende Kranz hätte dann in irgend einer

1) [Nachträglich glaube ich einen dieser Abschlußssteine nachweisen zu können. Ich hatte mix an Ort und Stelle notiert, dafi hoch oben auf dem jetzigen Ostende der Mantelmauer der Königsnische eine gerundete Platte aus Hag. Eliasstein liege, die durch die Ausgrabenden hier fälschlich aufgebaut sein müsse, da sie kleineren Durchmesser aufweise, als der übrige Wandbogen. Auf einer Photographie, die diesen oberen schmalen Raum darstellt, der durch die Grenzmauern von Lysanderkammer und Argos-Nische gebildet wird und auf dem Bulle-Wiegand ursprünglich das hölzerne Pferd aufstellen wollten, ist rechts ein Stück dieser Kranzplatte zu erkennen. 
Weise auch auf die Anten übergegriffen, da die jetzige Oberseite der WestAnte noch Auflager. zeigt.

Oben war die Dicke der Fundamentmaner auf etwa 1,80 veranschlagt, woron 0,32 aufen überstehender Rand ist. Man wird die Sockelstufen nicht genau ebenso tief, wie die unter ihr lagernde Fundamentstärke (c. $1,80-0,32 \Rightarrow 1,48$ gemacht, sondern wird sie innen etwas kürzer gehalten haben, etwa $1,40-1,30$. Nun ist die untere Sockelstufe der Argoskönige 1,25, die obere 0,82 tief, so daß unsere Fundamentdicke $(1,48)$ sehr gut zu diesen Maßen stimmt. Denn wenn man die Tiefe von Schicht $e$, die nach der auf Abb. 11 sichtharen Auflagerfläche auf der Oberseite von $d \mathrm{zu}$ schliefen, bis an den Aufenrand gereicht haben könnte, mit 1,40 oder mit 1,30 taxiert, wird man über ihr nur noch eine Stufe $(f)$ ansetzen wollen, die vorn c. $10-15 \mathrm{~cm}$ abtreppte. Hinten wäre $f$ von dem oben erwähnten Aukenkranz umschlossen worden, oder bätte wieder abgetreppt. Jedenfalls bewegte sich die Tiefe der Standplatten in dem normalen Maßs von c. $0,90-1 \mathrm{~m}$.

Endlich ist darauf zu verweisen, daßs nach $A b b .2$ die jetzige Oberkante des Hellenikó etwa in der Höhe unserer obersten Fundamentlage (unter Euthynteria a) verläuft. Ueber ersterer war noch eine abschliessende, dicke Quaderschicht, und dann noch einige flachere Deckquaderlagen nebst niedriger Balustrade zu vermuten (oben p. 79,1). Darnach würde $a$ der Abschlußlage des Hellenikó, $b-l$ seinen Deckquadern an Höhe entsprechen, so daß unser Paviment $d$ mit der Mauerkante (ohne Balustrade) in gleicher Höhe abschlob. Dies ist nun gerade die Höhe, die wir verlangen müssen, damit die dicht bis an das Hellenikó heranreichenden Epigonenstatuen nicht durch die Linie der Mauerkante geschnitten wurden. Der Sockel $e-f$ hätte dann der Höhe der Mauerbalustrade entsprochen oder, falls sie fehlte, die Statuen über die Abdecklagen der Mauer wirkungsvoll emporgehoben. Eine so hohe Aufstellung empfahl sich auch deshalb, damit die mittelsten, von der Straße fast $6 \mathrm{~m}$ abstehenden Statuen für den Vorbeigehenden nicht versanken.

Sehließlich muf an den Anten ein ähnliches Zurückbleiben der Standfläche $(f)$ von der Vorderwand gefordert werden, wie wir es bei den Königsstatuen der gegenüberliegenden Nische nachwiesen (Klio VII p. 397 u. 421, 1), wo die Statuenstufe je $2 \mathrm{~m}$ weit vor den Vorderecken aufhört. Denn unter keinen Umständen durften unsere Anfangs- und Endstatuen den auf der heiligen Strake sich Nähernden die Rüekseiten zukehren. Da nun die innere Peripherie von $e$, bei einem Radius von $(6,35-1,50 \Rightarrow 4,85$, etwa eine Länge von $15,25 \mathrm{~m}$ hätte, würde sich diese durch das Entferntbleiben von der Vorderkante um c. $4 \mathrm{~m}$, d. h. auf $11,25 \mathrm{~m}$ reduzieren; für $f$ vergrößert sich dieses Mał̇ jedoch wieder - wegen des durch die Abtreppung verlängerten Innenradius - auf c. 11,40-1,60 m. DarSie hat an der oberen Aufenkante einen schwach profilierten Ablauf, ähnlich den Platten unter den Orthostaten an den Rückwänden der Exedren, und wenn sie nieht zu einer derselben gehören sollte, - besonders die Exedra auf der Ostseite der $\ddot{\alpha} \lambda \omega \varsigma$ neben der großen Treppe käme in Betracht -, so bliebe nur das Epigonenhalbrund übrig, für dessen oberste,'Schichten man H. Eliasstein-Material voraussetzen darf]. 
nach käme auf unsere 8 Statuen eine Aufstellungslänge von fast $12 \mathrm{~m}$, c. $1^{1 / 2} \mathrm{~m}$ pro Statue. Das ist natürlich zu viel, - bei den Argoskönigen sind die Basensteine etwa halb so breit $(0,82)$ - und beweist, dak auch bei unseren Statuen $\mathrm{l}$ e e r e $\mathrm{Z}$ wis c h e n s t e in e vorhanden gewesen sein müssen. Im übrigen scheint das Epigonenhalbrund nur unwesentlich kleiner zu sein als das der Argoskönige, da jenes 12,70, dieses 13,74 Durchmesser hat (vgl. hierüber den Schluf des nächsten Abschnitts).

3. Zeit und Veranlassung der Epigonen-Errichtung.

Unmittelbar an die Schlufworte der Septem (Anfang von Abschn. 1 der vorigen Studie) schließst Pausanias folgenden Bericht:

"Von derselben Kriegstat aber, wie es mir scheint, weiheten die Argeier auch die von den Griechen sogenannten Epigonen. Denn auch von diesen stehen Bildsäulen da: Sthenelos und Alkmäon u. s. w. (s. unten)."

Daßs die Septem und die Epigonen von ein und derselben Kriegstat errichtet seien, bezeichnet der Perieget ausdrücklich als seine Vermutung. Sie gibt sich deutlich als Verlegenheitsauskunft zu erkennen und unterliegt den gröbten Bedenken ${ }^{1}$ ). An sich würden beide Gruppen als Pendants für einen Sieg gut passen, aber sie müften sich dann äuferlich als genau entsprechende Gegenstïcke ausweisen. Das Gegenteil ist der Fall. Die Septem standen auf einem einfachen Stufenbathron ohne Unterbau, sie sind, nach allem was wir annehmen müssen, ein Reihenmonument gewesen, ganz für sich geschaffen und durch die seitliche Aufstellung des Amphiaraos-Wagen kompletiert und abgeschlossen. Sodann trugen sie eigene Weiheinschriften, die sie als Denkmal für den Sieg von Oinoe bezeichneten und die Künstlersignatur des Hypatodoros und Aristogeiton enthielten. Die Epigonen dagegen zeigen die ungewöhnliche Forn des Halbrunds und ruhen auf einem gewaltigen, tief fundamentierten Unterbau; sie schienen ihre besondere vollständige Weiheinschrift zu haben, die den Namen des Sieges leider nicht nannte, aber keinerlei Künstlernamen, - wenigstens stand dieser nicht an so sichtbarer Stelle, daf ihn Pausanias sich notierte. Anch können sie darum nicht gut das Gegenstück zu den Septem gewesen sein, weil ihnen selbst ein richtiges Gegenstück gegenübersteht in Gestalt des Königshalbrunds.

Haben wir also zwei völlig verschiedene Anathemata zu erkennen, so

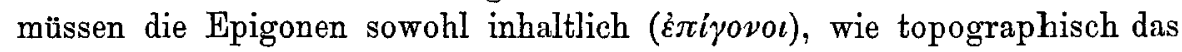
jüngere der beiden sein. Anderseits schienen sie älter, als das hölzerne Pferd, da dieses zwischen Septem und Marathonmonument eingeschoben werden mufte, weil jenseits der Septem bereits alles besetzt war. Dar-

1) Vgl. meine Bemerkungen Archäol. Anz. 1902, S. 19 Anm. 7, wo jedoch die topographischen Indizien durch die Eliminierung der 'Sockelmauern' (oben p. 74 f.) jetzt fortfallen. Auch Homolle hatte Zweifel an der Zusammengehörigkeit dieser ArgiverAnatheme (Septem, Amphiaraos-Wagen, Epigonen) gehegt, Bull. 21, $300\langle 400\rangle$. 
nach würde man die Epigonen der Zeit zwischen 456 und 414, genauer etwa den drei Dezennien 450-420 zuweisen wollen, und ich sah eine Bestätigung hiefür in dem Schriftcharakter der grofien Weiheinschrift, der für jene Jahre vorzüglich paßite; nur mußite man in Rücksicht auf das $\oplus$ der oberen Grenze dieses Zeitraums möglichst nahe bleiben.

Nun ergab aber die Durchmusterung der älteren Geschichte von Argos absolut kein Ereignis, das als Anlafi zu einer so grofen Dedikation betrachtet werden konnte. Denn schon im J. 450/49 wird zwischen Argos und Sparta ein 30jähriges Bündnis geschlossen, das 420/19 zu Ende geht. Während dieser ganzen Zeit ist kein größerer argivischer Krieg bekannt, wohl aber wissen wir, daß Argos auf das strengste neutral bleibt, wohlwollend neutral nach beiden Seiten, Athen und Sparta; es treibt mit beiden Handelsgeschäfte, liefert Waffen und Kriegsbedarf und kommt zu Wohlstand und Ansehen ${ }^{1}$ ). So schien in historischer Hinsicht nur die Annahme übrig zu bleiben, daßs wir hier gar kein Siegesanathem vor uns hätten, sondern daßj die Argiver dieses prachtvolle Weihgeschenk $\dot{\varepsilon} \xi \dot{\varepsilon} \pi \dot{i}-$

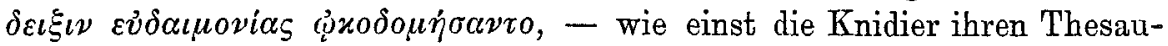

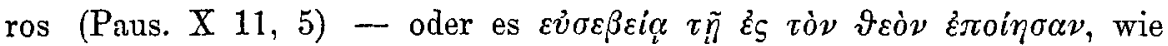
die Potidaeaten den ihrigen (ebda. X, 11, 5). Aber freilich kannten wir solche Ursachen bisher nur bei der Erbauung von Schatzhäusern; denn die gewöhnlichen Staatsanatheme verdanken ibre Entstehung fast ausnahmslos kriegerischen Anlässen.

Nicht besser erging es in epigraphischer, archäologischer, architektonischer Hinsicht. Die große Weiheinschrift gehörte dem Schriftcharakter nach so genau in die Zeit der Septem und der Schlacht von Oinoa (456), daß3 ein zweites fast gleichzeitiges Anathem, das doch im Aufbau und Anordnung so total von den Septem verschieden war, äußerst befremdlich und unerklärlich erschien. Sodann wollte die Heldenliste der Epigonen gar nicht zu derjenigen der Septem passen und wies sich als einer andern jüngeren Quelle entstammend aus, sodaß an eine zeitliche Nachbarschaft beider Anatheme nicht mehr zu denken war. Endlich lief sich die angebliche Inschriftstufe in die Architektur des Halbrunds absolut nicht passend einfügen, um so weniger, als weder ihre Oberseite noch die der Unterstufe abgetretene Flächen aufwiesen, also keine Trittstufen gewesen sind.

So wirkte es als Befreiung, daß schließlich Bulle die Weiheinschrift vielmehr dem Stufenbathron der Septem zuerteilte, - aber die Datierung des Epigonenhalbrunds schwebte nun völlig in der Luft, da wir weder von dem Anlaf3 der Stiftung, noch von dem Künstler, noch von der Weiheinschrift, noch von der ungefähren Zeit das Geringste wissen. Es blieben als Anhaltspunkte nur die ungewöhnliche Form des Halbrundbaus und der

1) Ueber diese Verhältnisse und den gegen Frühjahr 449 geschlossenen 30jährigen Frieden vgl. Busolt III, 1, 339; III, 2, 856; 1122; 1189. 
Name der Argiver als Stifter; daneben als sehr wahrscheinlicher Anlak ein Sieg über Sparta.

Betreffs der $R$ u $n d f$ or m lieken sich folgende Erwägungen anstellen. Abgesehen von dem anders gearteten, nur flach gerundeten Bathron der 9 achäischen Helden, die Nestor um den Zweikampf mit Hektor losen läßst (Gruppe des Onatas zu Olympia), schien unser Bau das früheste Beispiel des streng geometrischen Halbkreisbathrons ${ }^{1}$ ). Seine Erfindung dürfte auf einen architektonisch und bildhauerisch gleich geschulten Künstler zurückgehen, der nicht nur die Unübersichtlichkeit und Langeweile der geradlinigen Reihenmonumente beseitigen wollte ${ }^{2}$ ), sondern der auch die harmonische Wirkung der Rund- und Halbrund-Bauten in Theorie und Praxis genau kannte und sie auch für Skulptarwerke in Anwendung brachte. Es erscheint mir kein Zufall, dati gerade ein argivischer Künstler die eben geforderten Eigenschaften in höchstem Maße besaf. Der jüngere Polyklet ist zugleich Architekt und für Rundbauten das größte Genie gewesen, das jemals gelebt hat ${ }^{3}$ ). So glaubte ich mit einer gewissen Wahrscheinlichkeit, ihn als Künstler der Epigonen vorsclılagen zı dürfen, um so mehr, als auch sonst Gruppenkompositionen von ihm bekannt sind, z. B. die Gruppe von Apoll, Artemis, Leto im Heiligtum der Artemis Orthia auf dem Berge Lykone bei Argos. Dafi Pausanias diesen Künstlernamen verschwiegen hat, fiele wenig ins Gewicht, denn er tut dasselbe auch bei dem gegenüberliegenden Königshalbrund, wo wir den Namen des Antiphanes ebenfalls erst aus der Inschrift kennen lernten (Klio VII, 406 ff.).

Auch Bulle kam betreffs des jüngeren Alters des Halbrunds zu ähnlichen Schlüssen: 'Was wir an Halbkreisbasen älterer Zeit besitzen (Ona-

1) Ueber das Bathron des Onatas handelt Sauer, Statuar. Gruppe p. 38 f.; es war wie die Situationspläne zeigen, nur ganz flach gerundet. 'Das wirklich halbkreisförmige Bathron der Memnon-Achilleus-Gruppe des Lykios steht vereinzelt' (Sauer 39, Anm. 139). Es ist kleiner (etwa 5-6 m Durchm.) als unseres, auch ist sein Unterbau oblong, nicht rund.

2) „Die Rundung des Bathrons erlaubte es, die Blickrichtungen der neben einander stehenden Figuren nach einem Punkte konvergieren zu lassen, ohne dafs die Figuren selbst ihre en-face-Stellung hätten aufgeben müssen ${ }^{*}$. Sauer a. a. 0. 38.

3) Er hat nicht nur die Tholos von Epidauros gebaut, - das Vorbild der nur wenig jüngeren delphischen im Pronaiatemenos - , sondern auch das daneben liegende Theater, von dem Pausanias (II 27,5) bekanntlich sagt: , es erscheint mir auf das höchste sehenswürdig; denn an Pracht übertreffen die römischen Theater weit alle andern der Welt, an Größse aber (alle) das der Arkader in Megalopolis; welcher Architekt aber wäre imstande, sich in Bezug auf Harmonie und Schönheit mit Polyklet zu messen?" Und ein ebenso wenig zufälliges Zusammentreffen dürfte es sein, wenn das Halbrund der Argoskönige bei einem so feinsinnigen Kenner wie Bulle die lebhafte Erinnerung und dasselbe Empfinden auslöste, wie das Halbrund des polykletischen Epidauros-Theaters (vgl. Klio VII 421 f.). - Auch daf ich gerade meine Worte "von der Harmonie und Schönheit" des Argoshalbrundes (Klio VII 398) jetzt im Pausaniastext bei dem Polyklet-Theater wiederfinde, ist merkwürdig.

131 
tas- und Apolloniatenbasis in Olyrapia, Ergebnisse Bd. II p. 146; Bd. V Nr. 692; Kononbasis auf der athenischen Akropolis, Michaelis, Arx Athen. tab. XXXVIII 15), sind einfache Stufenbasen ohne Pflasterung des Innenraums. Für die Anlage eines Pflasters ergibt sich bei ihnen nicht die geringste sachliche oder künstlerische Veranlassung ${ }^{1}$ ). Sodann sieht die rustika-artige Behandlung der Quader am rechten (westlichen) Ende gar nicht nach dem 5., sondern durchaus nach dem 4 . Jahrhundert aus, das solche technische Formen als künstlerische zu verwerten beginnt' ${ }^{2}$ ).

Diesen Indizien wollte aber der Argeier-Name als Stifter schlechterdings nicht entsprechen. Denn so wenig die ältere Geschichte von Argos (460-420) eine passende Stelle geboten hatte für die Unterbringung eines so groken Sieges über Sparta, so wenig taten es die Jahre des 'mantineiischen' Krieges (420-414) oder die folgenden, oder die jüngere argivische Geschichte im IV. Jhdt. Denn die 3 großen Siege 456 (Oinoa), 414 (Thyrea), 369 (Eroberung Lakoniens zusammen mit Epaminondas) hatten bereits ihre Anatheme. An einen anderen Gegner als Sparta aber zu denken, - etwa an die drei Feldzüge gegen Epidauros ${ }^{3}$ ) - verbietet sich aus äußeren topographischen und aus inneren historischen Gründen; wir kennen keinen passenden, glänzenden Argeier-Sieg über andere Feinde. „Eine historische Veranlassung für die Weihung der Epigonen läßst sich in der Mitte oder 2. Hälfte des IV. Jahrhunderts zweifellos ebenso gut oder besser ebenso schlecht finden, wie in der 2. Hälfte des fünften", sagt darum Bulle mit Recht, aber wenn er schliebt: 'Völlig verständlich wird uns die Epigonenbasis mit einem Schlage, wenn wir sie als eine wirkliche Epigonin, als Nachahmung der Königsnische erkennen', so glaube ich, daß dieser Schluß zu weit geht.

Um es kurz zu sagen: die Epigonen sind weder älter noch jünger als die Argosnische, noch sind sie überhaupt ein getrenntes Pendant von ihr, sondern beide Halbrunde bilden ein einziges Weihgeschenk, dessenzusammengehörigeHälftensie

1) In jüngere Zeit gehören die beliebten halbrunden Exedren mit Sitzbünken, die in Delphi, Delos und Priene mehrfach [auch im Traianeum zu Pergamon], in Epidauros sehr häufig vertreten sind. Hier war natürlich die Pflasterung des Innenraums zweckmäfig, ja unentbehrlich. $B$.

2) Fine übersehene Notiz, die erst während der Korrektur bemerkt wird, gibt in meinen Zeichnungen für das Epigonenfundament Z-Klammern an, was für eine Datierung in das 5. Jahrhundert sprechen würde. Jedoch sind die Gründe für das 4. Jhdt., wie sie von Pomtow oben weiterentwickelt', werden, so zahlreich und schwerwiegend, daßs wir die Frage der Klammerform bis zu einer Nachprüfung des ganzen monumentalen Bestandes außer Betracht lassen müssen. B.

3) Ueber die Verhältnisse von Argos nach Ablauf des 30jährigen Friedens (420) siehe Busolt III, 2, 1206, 1216, 1227 ff.; über die Züge gegen Epidauros ebda. p. 1233 ff. 1251 ; über die Oligarchenherrschaft und den neuen 50jährigen Vertrag p. 1253 ff.; ferner 1265 u. 1270. - Auf ältere Argiver-Siege über Korinth deuten die Inschriften Olympia V Nr. 250 u. 251, aber sie gehören der Schrift nach in die Mitte des V. Jhdts. 
sind, errichtet aus der Beute der Verheerung Lacedämons im Jahre 369 v. Chr. Diese Erkenntnis zieht die weitere nach sich: daß die Architektur beider Halbrunde vielleicht von dem jüngeren Polyklet herrühren kann, während die $\mathrm{Statu}$ e $\mathrm{b}$ eid e m al von dem berühmtesten damals lebenden einheimischen Künstler verfertigt wurden, vo $n$ d e $m$ g reis e n Anti$\mathrm{ph}$ an es. Diese Lösung gelang erst, als alles andere versagte und die unglücklichen Epigonen mit einem non liquet entlassen werden sollten. Sie empfiehlt sich durch ihre Einfachheit, innere Wahrscheinlichkeit, äußere Unanstößigkeit, wie die folgenden Bemerkungen dartun mögen.

Man hat bisher angenommen, das Halbrund der Epigonen sei der König'snische zwar ganz ähnlich, aber doch von kleineren Dimensionen. Diese Differenz ist jedoch nur eine scheinhare. Sie ist hervorgerufen durch den Umstand, daß der Nischenmantel nicht unmittelbar hinter den Standplatten emporgehen durfte, sondern daß aus künstlerischen Rücksichten ein Zwischenraum $(0,31)$ gelassen wurde, der die Statuen von der $W$ and etwas abrückte. Dieser Zwischenraum war bei den freistehenden Epigonen unnötig und fiel darum weg; ihr Statuensockel, bezw. die Standfläche konnte bis an die Kreisperipherie heranreichen und sein Aubenrand lotrecht über dem der Orthostaten abschneiden. Auf diese Art gemessen wird der Durchmesser der eigentlichen halbkreisförmigen Basis, d. h. der Standfläche der Statuen in beiden Halbrunden genau gleich ${ }^{1}$ ).

Allerdings gilt dieses Maßs nur an der Vorderkante. Die Tiefe des Epigonenhalbrunds scheint geringer gewesen zu sein, etwa nur 6,05, sodaß die Peripherie keinen genauen Halbkreis bildete, sondern etwas abgeflacht war. Das könnte befremden, erklärt sich aber durch die Nähe der Temenosmaner (Hellenikó) einerseits, die Strafenbreite andererseits. Letztere durfte augenscheinlich nicht schmaler werden als $5 \mathrm{~m}$; so half sich der Künstler, indem er den Unterbau hinten gegen das Hellenikó stiek und die Rundung von der Halbkreisgestalt etwas abweichen ließs. Auch das Theater in Epidauros ist kein genauer Halbkreis. Für das Auge war die Abweichung in Delphi kaum wahrnehmbar, sie kann uns lehren, daß ursprünglich völlige Uebereinstimmung in den Maßen der Argos-Halbrunde

1) In der Königsnische beträgt der senkrechte Halbmesser bis zur Außenperipherie der oberen Sockelstufe 6,87-0,31=6,56 (K/io VII, 397). Den vorderen Durch-

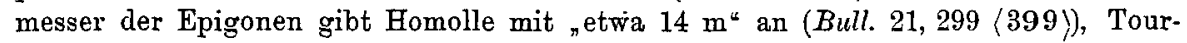
naire zeichnet ihn gut $13 \mathrm{~m}$ lang, wir maßen 13,10 Aufenkante, 12,70 innerhalb der Einbettung. Es ist mir aber nachträglich nicht sicher, ob bei der uns damals noch unklaren Struktur des Ganzen mein Helfer das Bandmak nicht etwa am Innenrand des Orthostats der Westecke angelegt hat, statt am Aubenrand. Das ist darum leicht möglich, weil die Euthynteria dort ganz im Boden steckt, während sie im Osten mit 0,32 Breite über die Orthostaten hinausragt und weil 12,70+0,32 annähernd unser sog. Aufenmaf 13,10 ergibt. Es ist daher wahrscheinlich, daf wir zu 12,70 noch die Orthostatendicke $(0,40)$ hinzuzuzählen haben, so daß wir mit dem Außenkranzhalbmesser auf $13,10: 2=6,55$ kommen, also genau soviel, wie der Königssockel hat $(6,56)$. 
Studien zu den Weihgeschenken und der. Topographie von Delphi. 315

beabsichtigt war, aber durch die gegebenen Raumverhältnisse etwas modifiziert wurde. Denn sonst hätte man gewiß unser ganzes Denkmal kleiner gehalten - seiner geringeren Statuenzahl entsprechend - und hätte seine Vorderkante nicht genau ebenso lang gemacht wie den Sockeldurchmesser des Gegenübers. Indessen muß betont werden, daß eine genane Neuvermessung der Reste dringend notwendig ist und vorher nichts Abschliessendes erreicht werden kann ${ }^{1}$ ).

Das Material ist in beiden Halbrunden gleich, - grauer Parnakstein für die grobe Architektur: Unterban und tragende Oberbanteile bei den Epigonen, Nischenmantel nebst Bekrönung, Roste und Anten bei den Königen ${ }^{2}$ ). Die feineren Architekturteile sind aus Hag. Eliasstein, wie Paviment, Vorderwand und Statuensockel der Könige beweisen. Seit die Inschriftstufen den Epigonen genommen sind, ist dort von diesem Material noch keine Quader nachgewiesen, aber dafs die genannten Teile auch hier aus ihm bestanden, ist nach Analogie aller anderen Anathemata des V. u. IV. Jahrhunderts zweifellos.

Auch der befremdliche Umstand, dak die Argiver als Gegenstand des nächsten Anatherns nach den Septem nicht deren Nachfolger, die Epigonen, gewählt hätten, sondern auf diese Ergänzung ihres Heroenkreises erst viel später verfallen wären, nachdem inzwischen das hölzerne Pferd als Siegessymbol geweiht war, - läßt sich jetzt exklären. Bei der Anlage des groken, aus zwei Halbrunden bestehenden Siegesanathems über Sparta wollte man sowohl dureh diese ganze schöne und gewaltige Architektur die große Nauarchoikammer nebenan ausstechen, als auch durch die Zahl der Heroenstatuen die vielen Schiffskapitäne Lysanders paralysieren. Man griff daher wiederum auf die mythische Zeit zurück und vervollständigte die Darstellung der argivischen Sagenkreise, aus denen fast 100 Jahre zuvor die Septem entnommen waren, nach oben und unten; der Stammvater Danaos und seine Nachkommen bis auf Herakles zeigten den Ursprung und Verlauf der Urkönigszeit, die Epigonen den Abschluß des zeitlich und menschlich den' Lebenden näher stehenden Heldenzeitalters. Damit war die Zahl der spezifisch argivischen Sagenkreise erschöpft, es dürfte schwer sein, noch einen weiteren zu finden, der eine ähnliche große Teilnehmerzahl von je 8-10 Gestalten aufwiese. Natürlich setzte man die Epigonen neben ihre Väter auf die linke, die Könige also gegenüber auf die rechte Straßenseite.

Im übrigen scheint es auch ohne Beispiel zu sein, daf älteren Anathemen erst später genaue Gegenstücke gegenübergestellt wurden; die antiken Künstler waren weder so erfindungsarm, noch klebten sie so am Anknüpfen

1) Ich habe darum die vorher vorausgesetzte hintere Abtreppung der ergänzten obersten Sockelstufe $f$ hier aufer Ansatz gelassen.

2) Daßs bei letzteren unter der Osthälfte der Vorderwand eine Lage Konglomeratstein verwendet ist (Klio VII, 399), dürfte belanglos sein.

Klio, Beiträge zur alten Geschichte VIII 3|4. 
an frühere fremde Schöpfungen, daß̊ sie nicht für einen späteren Sieg ein neues selbständiges Anathem geschaffen hätten, individuell und losgelöst von den früher aufgestellten ${ }^{1}$ ). Heut ein großes schönes Halbrund weihen und ihm dreiß̈ig, vierzig Jahre später ein ähnliches gegenüberstellen, dürfte dem modernen Geiste näher liegen als dem antiken.

Ferner ist die griechische Geschichte des IV. Jahrhunderts, - im Gegensatz zu der des fünften - so gut bekannt, daßs uns sicherlich ein zweiter großer Argos-Sieg über Sparta überliefert wäre, wenn er überhaupt stattgefunden hätte. Aber wie unmöglich ein solcher Sieg in den Zeitläuften von 368-357, oder gar während des phokischen Krieges (357 bis 345), wo Delphi gesperrt war, oder in Philipps und Alexanders Zeit erscheinen würde, braucht nicht ausgeführt zu werden. Auch daß man nach der Mitte des IV. Jahrhunderts noch Reihenanatheme mythischer Helden geweibt hätte, dürfte undenkbar und ein Anachronismus sein.

Endlich läßt sich die topographische Schwierigkeit, daßs die Stelle der Epigonen unmöglich bis vor oder nach der Errichtung der Königsnische leer geblieben sein könne, vermittelst eines Hinweises erklären, der Bulle verdankt wird. Er vermutet, 'daß sehr wohl kleinere Sachen hier gestanden haben können, die etwa in den unruhigen Zeiten des 4. Jahrhunderts zu Schaden gekommen sein konnten oder die man den Argivern zu liebe entfernte'. Wenn dies auch im Hinblick auf den phokischen Krieg und in der Annahme einer späteren Epigonen-Errichtung gesagt war, so paßt es doch gut für die jetzt veränderte Sachlage. Man wird das hölzerne Pferd im J. 414 aus Platzmangel zwischen Septem und Marathonmonument steilrecht eingeschoben haben, weil die spätere Epigonenstelle durch kleinere ätere Weihgeschenke besetzt war; wahrscheinlich sind das ebenfalls argivische gewesen und zu ihnen dürfte der alte Argiverstein Homolles mit der angeblichen Aufschrift $\Delta o\left[\dot{v} \varrho \varepsilon \iota_{0}\right.$ i $\left.\pi \pi \sigma_{\varsigma}\right]$ gehören (s. unten). Auch weisen die $5 \mathrm{~m}$ Abstand, die unser Anathem heut von den Tarentinern trennen, darauf hin, daf an dieser Stelle jedenfalls kein besonderer Platzmangel geherrseht hat, als die Epigonen errichtet wurden ${ }^{2}$ ).

1) Etwas ganz anderes ist es, wenn derselbe Künstler an verschiedenen Orten später Repliken für neue ähnliche Taten verfertigt, wie Paionios die MessenierNike in Delphi und in Olympia.

2) Die Wabl des Platzes für dás Pferd möchte Bulle lieber so erklären, „dás der Ostrand der Argiver-Terrasse für einen Kolok sehr viel geeigneter war, als ihr westliches Ende. Das Rof flankierte so den Terrassen-Anfang, es bildete eine Art Rahmen und Abschluß für sie und wuchs"gewissermafen aus der Oertlichkeit heraus, während es weiter westlich, ohne struktive Anlehnung an die Geländeformation und zwischen kleineren Figuren, leicht wie ein plumpes Einschiebsel hätte wirken können. Die Wahl seines Ortes läßjt sich also aus künstlerischen Erwägungen vollkommen rechtfertigen und ich möchte diese der Vermutung von dem 'Platzmangel' vorziehen. Denn so gut später für die Epigonen Platz geschafft wurde, hätte es auch schon für das Pferd geschehen können, wenn der Künstler diesen Platz als den günstigsten ver- 
Daß schließlich Pausanias bei den Epigonen den Künstlernamen verschwiegen hat, ist uns jetzt verständlicher als früher, weil es beidemal demselben Künstler galt, dem Antiphanes.

Zu Vorstehendem macht Bulle folgende Bemerkungen: „Pomtows Vorschlag, die Epigonen als gleichzeitig mit der Königsnische anzusetzen, halte ich für eine sehr glückliche Lösung, da sie meiner Ueberzeugung vollkommen gerecht wird, daf dieses Anathem aus basengeschichtlichen Gründen im fünften Jahrhundert unwahrscheinlich, hingegen im vierten und im Zusammenhang und als Gegenstück der Königsnische völlig verständlich ist. Freilich lälb̈t sich diese Lösung zunächst nicht über den Grad einer 'sehr wahrscheinlichen Vermutung' hinausheben, doch werden hoffentlich neue Untersuchungen der Steine, die wir nach den vorliegenden Studien jetzt mit ganz neuen Augen betrachten würden, zu absoluter Gewißhheit führen.

Hingegen kann ich mich der Vermutung, dá der jüngere Polyklet der Schöpfer des architektonisehen Teils der Königsnische und der Epigonen sei, nicht anschließen, so ansprechend auch der Vergleich mit den Rund- und Halbrundbauten des Künstlers zuerst erscheinen mag. Aber die Hypothese hat keine rechte Unterlage - der jüngere Polyklet ist meines Wissens niemals in Delphi beschäftigt gewesen -, und sie nimmt dem Antiphanes unnötigerweise ein Blatt aus seinem Ruhmeskranz, das wir ihm nach unserer bisherigen Kenntnis durchaus zusprechen müssen.

Die Königsnische ist, wie wir früher sahen, im Grunde eine Erfindung der Not, weil der Raum erst dem Berge abgewonnen werden mußte. Der Künstler der Lysanderkammer war mit diesem System vorausgegangen. Antiphanes hatte den Gedanken, das steife Rechteck durch das lebendige Halbrund zu ersetzen und sodann durch die Vollendung zum Vollkreis ein Raumgebilde von ganz neuartiger Geschlossenheit zu schaffen. Aber dies architektonische Raumgebilde ist nur ein Rahmen, herumgelegt um ein ganz neues Schema der plastischen Komposition, das man die "Kreisgruppe" nennen könnte. Der Künstler wollte den Beschauer mitten in die Versammlung der Helden hineinstellen, nicht an ihr vorbeimarschieren lassen wie bisher, - um so das alte, hundertmal wiederholte Problem der Gesellschaftsgruppe mit neuem künstlerischem Leben zu erfüllen. So läfat sich die "Kreisgruppe" vollständig als die Erfindung eines Plastikers verstehen, der eine architektonische Umrahmung hinzunimmt, weil sie durch die Besonderheit der Oertlichkeit verlangt wird. Daf er dazu von einem jüngeren argivischen Genossen wie Polyklet, mag dieser auch vielseitiger und namentlich architektonisch tätig gewesen sein, eine Anregung oder gar werktätige Hilfe nötig gehabt hätte, halte ich für sehr unwahrscheinlich. Dazu ist die Basis und die Nische doch etwas viel zu Nebensächliches, und ihre Form ergibt sich zudem aus dem künstlerischen Grundgedanken der Kreisgruppierung fast von selbst.

langt hätte“. - Indessen konnte man sich 369 viel leichter entschließen, alte Weihgeschenke dem neuen Halbrund zu opfern, als 50 Jahre früher, wo jene wohl noch verhältnismä̈ig aktuell und neuer waren. 
Eine neue Erkenntnis ergibt sich nun für Antiphanes. Er, der so oft während seines langen Lebens in Delphi gearbeitet hat, wo die Art der Aufgaben (vielfigurige Anathemata) und die Oertlichkeit (Raumenge und starkes Gefälle) in Bezug auf die Aufstellung ganz besondere Anforderungen stellten, läfst sich nun immer deutlicher als der eigentliche Vollender der Reihen-oder Gesellschaftsgruppe erkennen. Während gewil beim marathonischen und sicher beim Lysanderweihgeschenk nichts als eine einfache Parataxe vieler, unter sich gleichartiger Statuen vorhanden war, hat Antiphanes zuerst bei den Arkadern, mitten zwischen dem Reihenschema seiner Mitarbeiter, nach Gruppierung gestrebt (Ath. Mitt. 1906, 487 ff. Nr. V, VI u. S. 491).

Daf die Gestalten der Königsnische unter sich in lebendiger Wechselbeziehung stehen, haben wir früher ansgeführt (Klio VII, 425 f.). Nun kommt neu hinzu, daß die rhythmische Melodie, die innerhalb der Nische ertönt, gewissermaken wie vom Echo zurïckgeworfen ein zweites Mal auf der linken Straßenseite sich erneuert. So ist für den Beschauer, der zwischen den beiden Halbrunden steht, das Ganze eine vollkommen geschlossene Welt von Formen, die durch die grobe simple Linie des Kreises zusammengefalit, durch die energische Axe der durchbrechenden Strabe aber wieder in zwei Hälften geschieden wird. In der verlorenen linken Hälfte werden wir sie von derselben wohlberechneten rhythmischen Gliederung der Einzelteile belebt denken mïssen, wie wir sie für die rechte Hälfte erschlossen haben und wie sie von Pomtow in Abschnitt 4 dargelegt wird.

Wir empfinden hier mehr als sonst, daßs unser Wissen Stückwerk bleibt. Denn wenn wir für Antiphanes den Ruhmestitel fordern, die trockene Sachlichkeit der Gesellschaftsgruppe als einziger mit rauschendem Leben erfüllt zu haben, so fehlte es eben all zu sehr an Vergleichsmaterial von anderen Orten, etwa in Olympia und Athen. Daß wir aber mit unserem Lobe wenigstens für Delphi recht haben, das mag ein Blick auf die Gruppe der thessalischen Tetrarchen zeigen, die wir später zu besprechen haben. Obwohl dort kein geringerer Name als der des Lysipp mit einer der Gestalten in einer gewissen Beziehung steht, so zeigt ihr Gesamtanblick doch nichts, was diese Gruppe von den älteren rein parataktisch angeordneten irgendwie unterschiede. Die Helden stehen einer nehen dem anderen, ohne in eine formale oder gar geistige Beziehung zu einander zu treten, jeder eine Statue für sich, keiner sich als Teil eines höheren Ganzen gebend. Und so denke ich werden wir einstweilen grade Antiphanes als den größten Meister der Gesellschaftsgruppe rühmien dürfen." - Bulle.

\section{Die Statuen der Epigonen.}

Pausanias zählt folgende Helden auf (vgl. den Anfang von Abschn. 3): "Sthenelos und Alkmäon, der, wie es mir scheint, als der ältere vor (seinem

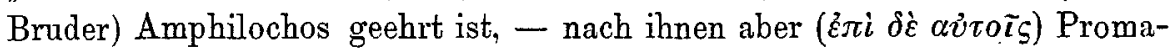
chos und Thersandros und Aigialeus sowohl als auch Diomedes; zwischen Diomedes aber und Aigialeus steht ( $\dot{\varepsilon} \sigma i \nu)$ Euryalos ". Jeder Unbefangene wird hieraus zunächst entnehmen, daß̉ nur 7 Statuen vorhanden seien; 
Studien zu den Weihgeschenken und der Topographie von Delphi. 319

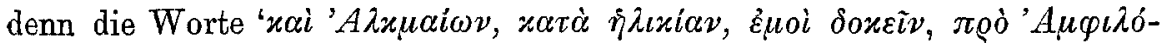

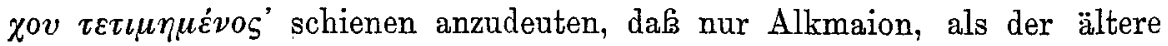
Bruder, der Ehre einer Statue gewürdigt sei $^{1}$ ). Indessen machte mich die in Abschn. 3 der vorigen Studie erläuterte Auslassung des Amphiaraos bei den Septem stutzig und die Vergleichung mit den übrigen Epigonenlisten ergab dann, daß ohne jeden Zweifel Amphilochos unter den Helden des zweiten Zuges subsumiert und hier dargestellt gewesen sein muk. Denn wir haben die Liste der Thebais (und der Tragiker) vor uns, die auch Apollodor (III 7, 2, 3) aufbewahrt hat, und die den Amphilochos stets ausdrücklich nennt. Die einzelnen Nachweise sind in Exkurs II gegeben.

Dab unsere Statuenreihe Gruppenbildung gehabt habe, schien sehr unwahrscheinlich, so lange man sie in die Mitte des V. Jahrhunderts setzte, wo die ungegliederte Parataxis die Regel war. Höchstens konnte an eine Zentralstellung des mittelsten Helden der anscheinenden Siebenzahl, des Thersandros, gedacht werden. Denn wenn wir bei der geradlinigen Marathon-Reihe die Hypothese der Zentralaufstellung einer Person (Miltiades) verwerfen muften (oben p. 85 u. 88,3) und sie auch sonst in geraden Reihen (Arkadern, Daochos) zu Delphi nicht nachweisbar ist, so war bei einem Halbrund das Verhältnis ein umgekehrtes: hier wird das Auge des Beschauers unwillkürlich und immer von Neuem auf die Peripherie-Mitte hingelenkt. Und Thersandros, des Polyneikes Sohn, bildete die Hauptperson des ganzen Zuges, dessen Ziel es war, jenen auf den Thebanischen Königsthron zu setzen, was nach der Sage voll erreicht ward.

Seitdem sich unser Anathem aber als viel jünger herausgestellt hat, kann man an einer Gruppen-Komposition nicht mehr zweifeln. Sie läßjt sich sogar in ihren Grundzügen deutlich erkennen, obwobl keine der Basen und nichts rom Sockel erhalten ist. Denn einerseits haben wir aus der Analyse der Argoskönige gelernt, wie die Gruppenandentung bei Pausanias noch durchzuschimmern pflegt, wenn man zwischen den Zeilen zu lesen vermag (Klio VII, 409), andererseits ist bereits erschlossen worden, dafi auch bei unserm Denkmal leere $Z$ wischensteine vorhanden gewesen sein müssen (s. den Schluß3 von Absch. 2), wodurch die Gruppengliederung eo ipso bewiesen wird. Darum wird selbst derjenige, der die Zugehörigkeit beider Halbrunde zu ein em Anathem noch nicht für sicher und deshalb die Künstlerschaft des Antiphanes bei den Epigonen nicht für bewiesen hält, doch a priori zugestehen, daß unsere Gliederung den jedenfalls ziemlich gleichaltrigen Königsgruppen ähnlich gewesen sei.

Nun sagt Pausanias, - um mit der letzten Gruppe zuerst zu beginnen, wie bei Gruppe $C$ der Argoskönige - am Schluf der Aufzählung:

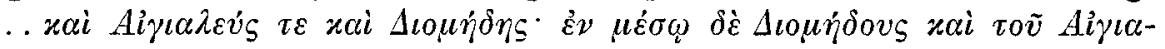

1) So nimmt z. B. Frazer (Paus. Bd. V p. 268) als sicher an, dak von den Amphiaros-Söhnen in Delphi nur Alkmäon dargestellt war, Amphilochos aber fehlte. 


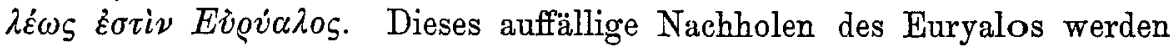
wir jetzt nicht mehr als stilistische Abwechselung betrachten dürfen, sondern ebenso wie die nachträgliche Nennung des Perseus bei den Königen als in der Gruppierung begründet erkennen: die drei Helden Aigialeus, Euryalos, Di omedes waren zu einer Gruppe vereinigt, die zwei äußeren lebhaft einander zugekehrt, der mittlere etwas zurückstehend.

Die nächste Gruppe muß die Mittel-Gruppe gewesen sein. So wie die Akrisios-Gruppe den Höhepunkt der Königsnische bildete und die Zentralstatue des Akrisios an Größe alle übrigen überragte, so wird, wie wir oben schon wahrscheinlich machten, Thersandros im Zentrum gestanden haben, als Hauptperson gröker als die andern, und zu ihm gesellt der Parthenopaios-Sohn Promachos.

Denn da man die nun folgenden Amphiaraos-Söhne Amphilochos und Alkmäon jedenfalls nicht auseinanderreißen darf, bleiben für die Anfangsgruppe nur diese zwei nebst $S$ then el os übrig. Es wäre auch möglich, dak letzterer ein wenig getrennt von dem Brüderpaar stand, dak also die Komposition mit ihm ebenso ruhig und schlicht anhob, wie drüben die längere Königsreihe mit Danaos und Hypermestra. Jedenfalls ist es kein Zufall, dass sich auf diese Art mühelos die symmetrischen Gruppen von $3+2+3$ Statuen ergeben. Sodann ist es sicherlich aus kompositioneller Absicht geschehen, daf die beiden einzigen Epigonen, die vor Troja gekämpft hatten: Diomedes und Sthenelos die Ehrenplätze an den Ecken erhielten. Wenigstens wird sich sonst kein Grund finden lassen, weswegen Sthenelos hier vor den anerkannten Führer des Epigonenzuges, Alkmäon, postiert wäre. Dafi zugleich der später vor Troja als 'König von Argos' kämpfende Diomedes an die Westecke gesetzt ward, ist geschehen, weil er hier allen Temenos-Besuchern, die rom Eingang herauf wandelten, zuerst in die Augen fiel. Er entsprach in seiner Stellung dem Abschluk der Königsreihe, dem Herakles.

So erhalten wir doch ein ungefähres Bild der Komposition: am Anfang und Ende die zwei berühmten Troja-Kämpfer Diomedes und Sthenelos - ähnlich wie beim Lysanderdenkmal je zwei Spartaner die Reihe der Kapitäne eröffneten und schlossen -, dann der Führer des Zuges nebst seinem Bruder; in der Mitte mit Parthenopaios die Hauptperson Thersandros; zum Schlufs die lebhafte Gruppe der drei letzten Helden, einschließjlich der Eckstatue des Diomedes. Auch bei diesem Denkmal hat also Antiphanes die Langeweile der Heldenreihe auf das glücklichste aufgelöst, und es ist wohl sicher, dak er auch hier neine Steigerung der künstlerischen Werte, eine Zunahme des Formenreichtums, eine Klimax in den Umriblinien" erzielt haben wird, wie sie von Bulle bei den Argoskönigen (Klio VII 425) so treffend nachgewiesen worden war. 


\section{- $\quad$ Exkurs II.}

Die Listender Septem und der Epigonen.

Ehe die Helden-Listen der beiden Weihgeschenke besprochen und auf ihre Quellen zurückgeführt werden, sei im voraus festgestellt, daß hier bei demal eine verschiedene Form der Sage zu Grunde liegt: denn nicht alle Helden des Epigonenhalbrunds sind Söhne der angeblichen Väter, der daneben stehenden Septem. Man vergleiche (Paus. X, 10,2):

Epigonen:

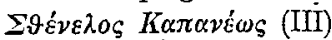

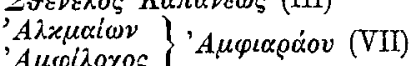

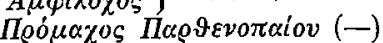

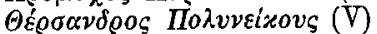

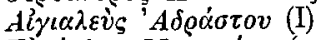

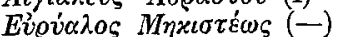

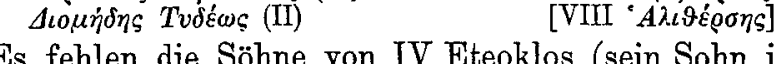
Septem:

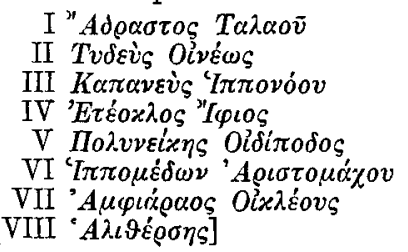

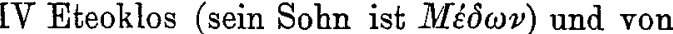

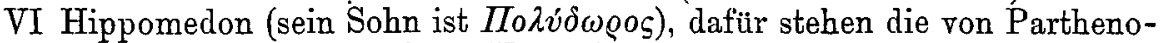
paios und Mekisteus da. Diese Verschiedenheit der Sagenformen war sehon Bethe aufgefallen und er hatte angedeutet, dak nach Roberts Meinung nes keineswegs sicher sei, dab diese zwei Statuenreihen einem und demselben Weihgeschenk angehörten" (Theban. Heldenlieder S. 110, Anm. 3; Robert, Hermes XXV 412, Nr. 2). Wir haben oben gesehen, daf diese Anatheme in der Tat auf ganz verschiedene Anlässe zurïckgehen und fast ein Jahrhundert auseinanderliegen dürften. Daher wird es jetzt verständlich, daß die Argeier im IV. Jhdt. einer anderen Version der Sage folgen und einen anders gearteten Epigonenkreis aufstellen konnten, der zu dem vor fast 100 Jahren gewählten Septemkreis nicht mehr recht stimmte. Umgekehrt wird durch diese Differenz bewiesen, dak wirklich ein langer Zwischenraum zwischen beiden Stiftungen gelegen haben muk.

$$
\text { Die Epigonen-Listen. }
$$

Zur Ermittelung der Quellen beider Sagenkreise empfiehlt es sich, von dem jüngeren Anathem, den Epigonen, auszugehen, da bei ihnen die Verhältnisse einfacher liegen. Die erhaltenen Epigonenlisten sind in der Tabelle zusammengestellt; es sind zwei statuarische Reihen A und $\mathrm{C}$ (in Delphi und in Argos) and zwei literarisehe B und D. Zu ihnen tritt eine fünfte $\mathrm{E}$, die aus den Namen der Epigonen-Väter, wie sie bei Aeschylus, Sophokles, Euripides als Teilnehmer am Septemzuge aufgezählt werden, abgeleitet ist. Aus dieser Gegenüberstellung ergibt sich sofort, daß je eine statuarische und literarische Liste zusammengehen, A mit B, $\mathrm{C}$ mit D. Der Hauptunterschied beider Klassen ist, daß in A-B Alkmeon als Führer des Zuges figuriert, was Apollodor III 7, 2, 1 und Diodor IV 66, 1 ausdrücklich bezeugen, während in C-D Aigialeus, der Sohn Adrasts, Anführer ist, was auch für E, den Tragikerkreis, Geltung hat, da Athene bei Euripides Suppl. $1216 \mathrm{ff}$. weissagt: "Aigialeus wird an seines Vaters Stelle die Epigonen führen und diese werden Theben erobern". Sodann fehlt in $\mathrm{A}-\mathrm{B}$ des Hippomedon Sohn Polydoros, in $\mathrm{C}-\mathrm{E}$ dafür der in

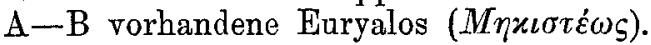

Nun geht, wie man längst gesehen hat (vgl. Bethe p. 88 ff.) die Tragiker-Liste der Septem auf die Thebais zurück, also weist die ihrer Söhne

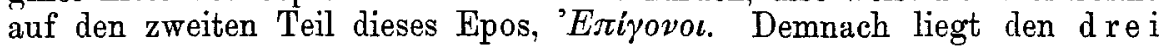
Listen C-E die Sagenform der Theba is-Epig onoi als Quelle 


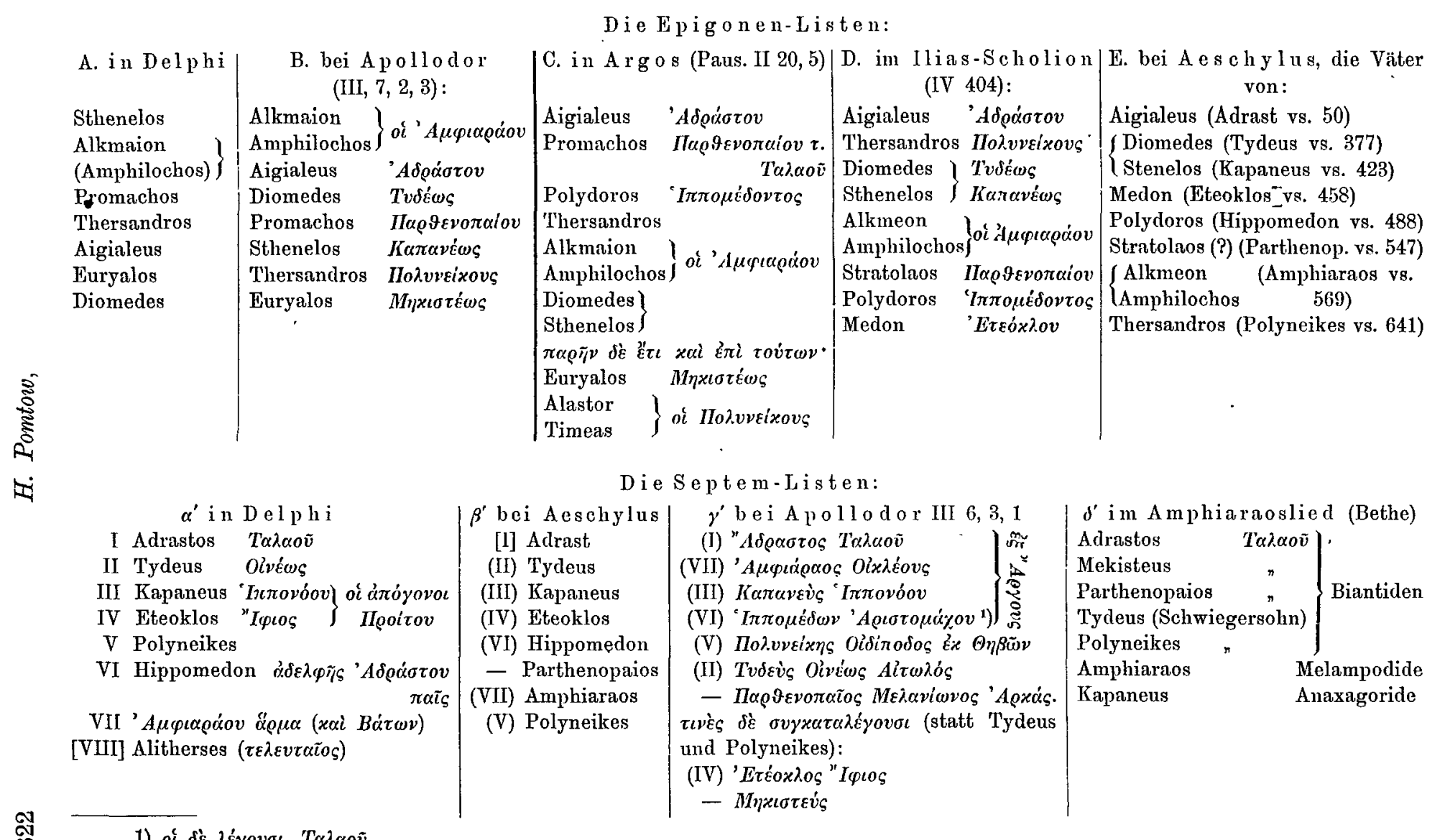


zugrunde, und ihre Version ist durch den allmächtigen Einflufo der Tragiker die herrschende geworden.

Dagegen hat für die Apollodor-Liste (B) Bethe sehr wahrscheinlich gemacht, dai sie, die den Führer Alkmeon voranstellt, auf die Alkmeonis

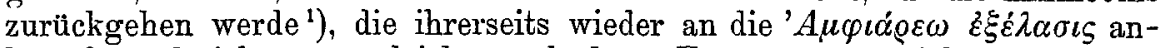
knüpfe, und viel später, gleichsam als deren Fortsetzung gedichtet sei. Denn Liste B setze diejenige Reihe der Väter (Septem) voraus, welche von Bethe vorher (S. 64) für das Amphiaraoslied aufgestellt worden war, 'indem aus diesem jeder Vater einen Sohn in jener findet und umgekehrt' (S. 112). Es dürfte darnach erwiesen sein, dafi die Argeier ihre delphische Liste A der Alkmeonis entlehnt haben, einem verhältnismäbig jungen, nach Wilamowitz erst um 600 gedichteten Epos, dessen Version schon Pindar Pyth. VIII, 40 gefolgt war ${ }^{2}$ ). Und da sowohl bei diesem, wie bei Apollodor beide Amphiaraos-Söhne am Zuge teilnahmen, so erhellt, dás wir soeben in Abschn. 4 mit vollem Recht den Amphilochos für die delphische Statuen-Reihe reklamiert haben, obwohl des Pausanias geschraubter Ausdruck dagegen zu sprechen schien. Die Anordnung der delphischen Statuen (Sthenelos als Pendant zu Diomedes usw., s. Abschn. 4). wich von der Anordnung in $B$ leicht $a b$, bringt aber sogleich nach der Eckfigur als erste den Anführer Alkmeon und seinen Bruder.

Die Liste $C$ beginnt Pans. II 20,5 mit folgenden Worten: 'nahe bei diesen Sieben - denn anch die Argeier sind der Dichtung des Aeschylus (nämlich betreffs der Sieben-Zahl) gefolgt -, stehen auch die (späteren) Eroberer Thebens : Aigialeus, der Sohn Adrasts usw.' und schliebt: 'es waren (stan-

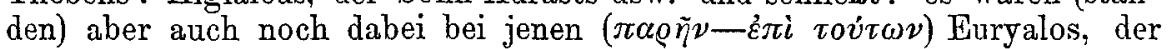
Sohn des Mekisteus, und des Polyneikes (Söhne) Adrastos und Timeas.' Hier hat Bethe (p. 110,4) nach Schol. Pind. Ol. II 76 mit Sicherheit Alastor hergestellt, und ich halte es für wahrscheinlich, dak die Argeier die Statuen dieser zwei sonst unbekannten Polyneikes-Söhne aus ihrer Lokaltradition hinzugefügt haben. Abgesehen von ihnen ist die Neunzahl der Epigonen die normale, denn bei den Tragikern war Adrastos, als Heerführer, nicht unter die Septem einbegriffen gewesen, und so kommt sein Sohn und der zweite des Amphiaraos zu der Zahl der sieben Söhne hinzu. Auffallend ist auch der Ersatz des Eteoklos-Sohnes Medon (Liste D) durch den aus Liste A-B beibehaltenen Euryalos ( $M \eta \varkappa \sigma \tau \tau \dot{\varepsilon} \omega \varsigma)$, den die Tragiker nicht kennen. Die Argeier scheinen also bei dieser Gruppe, deren Stiftungszeit ganz ungewiß bleibt, kontaminiert zu haben (Bethe p. 111): denn auch die Benennung des Parthenopaios-Sohnes als Promachos, - statt Stratolaos (in D) - geht auf A-B zurück. Sie könnten sogar zunächst die Liste der Alkmeonis, wie in Delphi, gewählt haben, machten jedoch ihren Königssohn Aigialeus zum Führer und fügten den Hippomedon-Sohn Polydoros hinzu, beides nach der Thebais, bezw. den Tragikern. Oder aber sie haben sich, worauf der Anhang der Polyneikes-Söhne deutet, nach Lokalsagen gerichtet, die uns unbekannt sind und die sich neben den Sagenformen der Epen und Tragödien im Lande selbst behauptet haben.

In Liste D gibt der Scholiast als Parthenopaios-Sohn den Stratolaos,

1) Bethe Theban: Heldentieder S. 112 und 117 (vgl. 130 und 134), im wesentlichen nach Immisch, Klaros S. 173-183.

2) v. Wilamowitz, Homerische Untersuchungen p. $328 \mathrm{ff}$. Zu Pind. Pyth. VIII vgl. Bethe p. 112, 7 und Immisch, Klaros p. 173. 
worüber vgl. Bethe p. 111 f., und bezeugt die Neunzahl der Epigonen ( $\dot{\varepsilon} \nu$ -

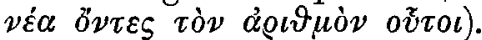

Liste E findet sich bei Aeschyl. Septem vs. 50 sqq., Sophokles Oed. Col. vs. 1310 sqq.; Euripides Suppl. 860 sqq. Dagegen schlieft die bekannte Aufzählung der Phoenissen vs. 125 sqq. den Adrast unter die Septem ein und läbt darum den Eteoklos aus.

$$
\text { Die Septem-Listen. }
$$

Schwieriger liegt die Sache bei den Septem, weil ihre in Argos errichteten Statuen von Pausanias nicht einzeln aufgezählt werden und die Listen an sich keine so große Verschiedenheiten aufweisen können, wie die der Epigonen. Die Abfolge der ersten Liste $\left(\alpha^{\prime}\right)$ der Tabelle schien mir zuerst auf einer Redaktion des Pausanias zu beruhen, der die 'Proetus-Abkommen' stilistisch-genealogisch zusammengeordnet habe. Aber Liste $\beta^{\prime}$ gibt sie genau ebenso und stimmt auch in der Reihenfolge der Helden I-IV und VII genau mit $\alpha^{\prime}$. Hierdurch wird zunächst erwiesen, daß in der Tat Amphiaraos (Nr. VII) seine Statue auch in Delphi hatte, weil die Erwähnung seines Wagens gerade an der Stelle in A stattfindet, wo in $\mathrm{B}$ er selbst steht. Die oben (in Abschn. 4 der Septem) hierüber aufoestellte Behauptung ist also bestätigt.

Sodann würde man, da soeben gezeigt war, daß diese Septem-Liste $\left(\beta^{\prime}\right)$ der Tragiker auf die Thebais zurückgeht, in welcher Adrast nicht mitgezählt war, sondern als Heerführer über den Sieben stand, ohne Weiteres auch die delpischen Statuen $\left(\alpha^{\prime}\right)$ auf die Thebais zurückführen können, wenn nicht in $\beta^{\prime}$ Parthenopaios erschiene, der in Delphi fehlte. Bethe weiß darum mit dieser Liste $\alpha^{\prime}$ nichts anzufangen, da der Ersatz des Parthenopaios durch Alitherses unerklärlich sei (p. 110, Anm. 3). Ich habe zuerst an eine Lücke im Pausaniastext gedacht, derart, daß zwischen

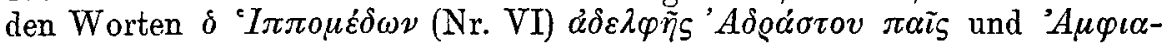

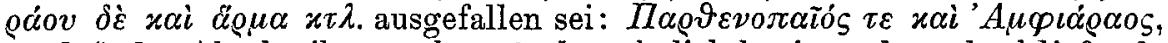
so dafi der Abschreiber nach $\pi \alpha i \bar{s}$ das ähnlich beginnende und schließende

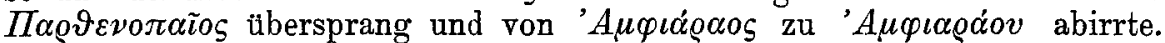
Wir würden dann nicht nur die genaue Liste, sondern auch die Abfolge der Thebais und der Tragiker erhalten bis auf den wohl erst durch Aeschylus absichtlich an die letzte Stelle verwiesenen Polyneikes. Und Alitherses als anscheinend neunter würde nicht stören, wenn das richtig ist, was oben im Abschnitt 3 der Septem über seine Zugehörigkeit zu dem Amphiaraos-Wagen gesagt war; denn darnach hatte er mit der Siebenzahl oder mit der Heldenreihe selbst gar nichts zu tun.

Ich möchte aber diesen Notbehelf der Lückenhypothese nicht für sehr wahrscheinlich halten, sondern glaube eher, dab die Argeier den bei den Tragikern, und darum wohl schon in der Thebais ausdrücklich für einen

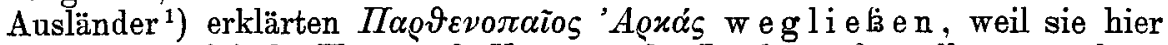
nur echt argivische Heroen als Vertreter des Landes aufzustellen wünschten und die Siebenzahl auch ohne ihn (und Alitherses) voll war. Und daß sie hier überhaupt der Thebais folgten, wird niemand wundern, der sich erinnert, wie dieses Lied dem alten Ruhm von Argos galt, - es begann

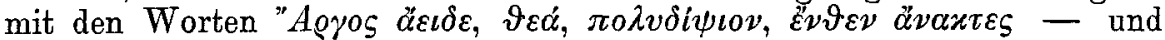
daf́ gerade damals, 12 Jahre vor der Schl. von Oinoe, Aeschylus die Tra-

1) Vgl. Eurip. Phoen. 1153 über Parthenopaios: of \& 'A

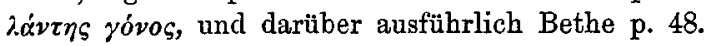




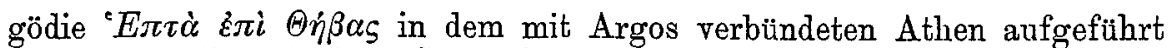
hatte (a. 468) und dieser Sagen-Stoff hierdurch gewissermaken aktuell und zum passenden Gegenstand argivischer Bildhauerkunst geworden war.

$\mathrm{Zu}$ dieser Rückführung unserer Liste $\alpha^{\prime}$ auf die Thebais wïrde es trefflich stimmen, dafi nach Bethe auch „die Darstellung des Abschiedes des Amphiaraos auf dem Kypseloskasten, die uns durch den Korinthischen Krater des Berliner Museums Nr. $1655 \mathrm{zu}$ lebensvoller Anschauung gebracht ist, die Sagenform der Thebais voraussetzt“ (p. 127). Denn gerade diese Szene haben wir oben (Abschn. 4) ebenfalls an unserm Anathem, neben den Septemstatuen zu erkennen geglaubt und haben ihre Hauptpersonen in der Wagengruppe des Amphiaraos, Baton und Alitherses wiedergefunden.

Nachdem so die Quelle unserer Anathem-Darstellung leidlich sicher nachgewiesen ist, kann ich mich den übrigen Listen gegenüber kurz fassen. Die des Apollodor $\left(\gamma^{\prime}\right)$ gibt die Namen von $\beta^{\prime}$, ordnet sie aber g e o g r a$\mathrm{p} \mathrm{h}$ i s c h und lägt den Eteoklos zunächst aus; aus letzterem Grunde mul sie indirekt aus den Phönissen stammen, von deren Liste wir wissen, daß sie in die mythologischen Handbüicher überging, aus denen auch Apollodor schöpfte. Der Eteoklos war dort hinterher aus der Liste des Aeschylus etc. angefügt, der Mekisteus aber aus der des Amphiaraos-Liedes $\left(\delta^{\prime}\right)$. Bethe hat diese Liste $\delta^{\prime}$ scharfsinnig rekonstruiert und erklärt (p. 63 ff.); sie enthält die Namen derjenigen Septem (aus den drei argivischen Eauptgeschlechtern), die als Väter zu der Epigonenliste (B) der Alkmeonis passen, welche in unserm Halbrund dargestellt und eben besprochen war.

Was endlich die in Argos aufgestellten Septem angeht, - für welche die Liste fehlt - , so ist es für uns von großem Nachteil, daß Pausanias sie nicht namentlich aufgezählt hat. Er sagt nur (II 20, 5): "Geht man von hier (dem Heiligtum der Horen) zurück, so stehen da die Bildsäulen des Polyneikes, des Sohnes des Oidipus, und derer, welche als Führer mit ihm vor den Mauern Thebens kämpfend gefallen sind. Diese Männer hat Aeschylus auf die Zahl von nur Sieben zurückgeführt, während doch mehr Führer sowohl aus Argos als auch aus Messene und auch einige Arkader zu Felde gezogen sind. Nahe bei diesen Sieben, de n n auch di e Argiver sind der Dichtung des A ischylos gefolgt, sind diejenigen aufgestellt, die (später) Theben eingenommen haben: Aigialeus etc. (Liste C)." Aber da Pausanias die Epigonen beidemal (in Argos und Delphi) einzeln nennt, kann der Grund jener Kürze nur der sein, dab die Argos-Gruppe in der Tat nur die aus Aeschylus altbekannten Namen enthielt, die der Periget aus stilistischen Gründen nicht wiederholen mochte, und diese

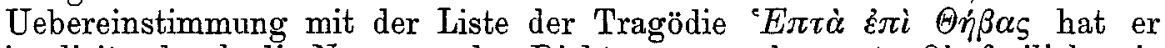
implicite durch die Nennung des Dichternamens bezeugt. Ob freilich, wie Bethe will, auker den 8 Namen der Liste $\beta^{\prime}$ die sicherlich in Argos standen, auch noch Mekisteus (nach dem Amphiaraos-Liede, $\delta^{\prime}$ ) hinzugefügt war, müssen wir dahingestellt sein lassen.

Als Resultat dieser Untersuchungen erhalten wir demnach, dak die Argeier im Jahr 456 v. Chr. den Inhalt ihrer Anathem-Darstellung aus dem Sagenstoff des altberühmten Epos Thebais entnahmen, der damals auch durch des Aeschylus 'Sieben gegen Theben' in den Vordergrund des Interesses gerückt war, - daß sie aber im IV. Jhdt. dem jüngeren, allmählig an Geltung gewinnenden Epos Alkmeonis den Vorzug gaben und die Statuenliste des Epigonenhalbrundes aus ihm entlehnten, obwohl sie zu den alten daneben stehenden Statuen der sieben Väter durchaus nicht mehr stimmte. 
Das untere Anathem der Tarentiner (Nr. 17).

Hinter der Beschreibung der Argoskönige macht Pausanias einen Abschnitt. Denn nach ihren. Schlubiworten ... 'und noch vorher bis zu Perseus' (vgl. Klio VII, 406) hebt er aufs neue an, wie folgt ${ }^{1}$ ):

"Von den Tarentinern aber stehen eherne Roße und gefangene Frauen da (aus der Beute) von den Messapiem, Barbaren die dem Tarentinischen benachbart sind; es sind aber Werke des Ageladas aus Argos. Tarent ist eine Kolonie der Lacedaemonier usw. Nahe bei dem Weihgeschenk der Tarentiner liegt das Schatzhaus der Sikyonier.. Die Knidier aber haben Bildsäulen nach Delphi gebracht usw.; diese stehen beim Thes. der Sikyonier."

Daß solch ein Abschnitt bei dem Periegeten hier und oft darauf deutet, dats er inzwischen auf die andere Strakenseite hinübergegangen ist, habe ich schon früher dargelegt (Archäol. Anz. 1895, 9) und es wurde neuerdings durch unsere Ansetzung des hölzernen Pferdes bestätigt, bei dem sich ebenfalls der Abschnitt und der stillschweigende Wechsel der Straßenseite vorfindet (oben p. 103). Da außerdem die ganze rechte Seite des Weges durch die Nischen Nr. 7-11 nebst Aitolerbasis (Nr. 12) und langer Stützmauer gefüllt ist (Klio VII, 427-444), die Tarentiner-Gruppe aber keinesfalls in einer dieser kleinen Kammern stand, bleibt nur der Raum südlich der Straße übrig, von den Epigonen bis zum Sikyonier-Schatzhaus.

Hier erhebt sich, $4,40 \mathrm{~m}$ westlich der Epigonen, eine in situ befindliche, jetzt allseitig freistehende pol y g o n e Ma u er-Ecke, die auf Abb. 13 (Taf. IV) wiedergegeben ist. Sie hat heut noch eine Höhe von 1,35, zeigt 3 Polygone übereinander, ist nach Osten zu augenscheinlich für Ansicht berechnet und hat hier jetzt noch eine Breite von $1 \mathrm{~m}$. Von der gegenüberliegenden viereckigen Nische (Nr.7) ist die Nordkante des Mauerstücks 5,55 m entfernt, vor letzterer hat man eine nicht zugehörige Platte angelehnt. Daks wir eine Ecke vor uns haben, erkennt man nur an der senkrechten Kante rechts, denn von der Nordseite ist nichts erhalten; vgl. auch Abb. 12 (Taf. IV), aus der ersichtlich ist, dab die Mauerblöcke innen (nach Westen zu) wie gewöhnlich nur roh behauen waren. Wir haben also den Rest einer von S. nach N. streichenden, n a ch 0 s t e n of fen liegenden Ter$\mathrm{r}$ a s s e $\mathrm{nt}$ tï $\mathrm{z} \mathrm{m}$ a u er vor uns, die einst c. $2 \mathrm{~m}$ hoch gewesen sein mag. da wenigstens noch eine Schicht anflag. Das Material ist Parnaksstein.

Erinnern wir uns einerseits an das oben p. 84 betreffs der sich überhöhenden Terrassenabsätze Gesagte, zwischen denen der heilige Weg wie

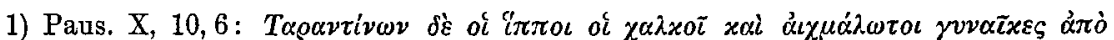

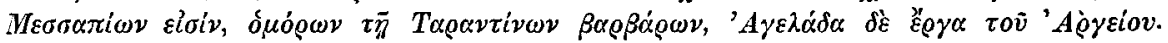

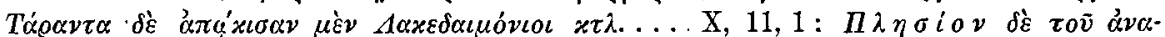

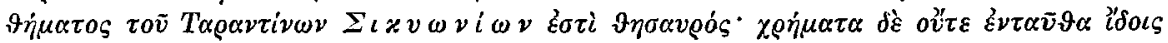

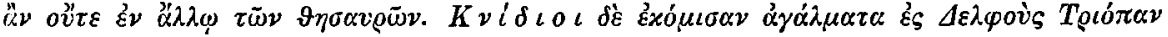

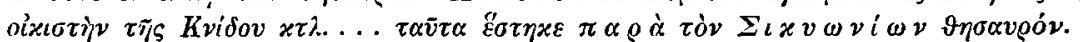


ein Einschnitt emporgeht, und andererseits an die im Anfang dieser Strtdien hervorgehobene Tatsache, daß Pausanias „nur grofe, in die Augen fallende Anatheme, Statuenreihen in geräumigen Kammern, auf gewaltigen

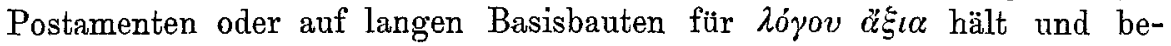
schreibt" (Athen. Mitt. 1906, 444), - so kann es nicht zweifelhaft sein, dabi wir in dem eben beschriebenen Rest die Ostseite der Tarentiner-Terrasse vor uns haben. Da die Strafe hier nicht mehr übermäßig steigt (vgl. die Abb. 16 auf Taf. II in Klio VII), so beweist die Höhe der Mauerecke, daßB das Anathem, das sie zu tragen bestimmt war, nicht nur sehr hoch lag, sondern auch weit nach Westen gereicht haben muk, also recht lang war. In der Tat hat die Strecke von der Ostseite unserer Mauer bis zur Ostwand des Sikyon-Thesauros eine Länge von $16,30 \mathrm{~m}$, deren größten Teil die Tarentiner eingenommen habén müssen. Im übrigen steht die erhaltene Ecke nur wenig von der Vorderflucht des Epigonenhalbrunds zurück und zeigt uns, dafs die Strafe von hier ab etwa $5,55 \mathrm{~m}$ Breite gehabt hat.

Nun wurden im Mai 1894 in dieser Gegend mehrere Quadern aus Hag. Eliasstein gefunden, die durch die Einlafiöcher als Standplatten von Statuen, bezw. Pferden kenntlich waren und an der Vorderseite in großen Buchstaben das Wort $\Sigma \Delta$ EKATAN trugen. Homolle bezog sie mit Wahrscheinlichkeit auf unser Anathem und ließs sie links vom Wege zusammengesetzt aufbauen ${ }^{1}$ ). Vgl. Abb. 14 auf Taf. IV. Die genauere Beschreibung der Platten wird am Schluß dieser Studie gegeben, während hier einige allgemeine Erwägungen folgen:

Der Schriftcharakter weist auf das V. Jhdt., wahrscheinlich auf dessen erste Hälfte $\left.{ }^{2}\right)$; genau in letztere Zeit gehören auch die Klammern (1-),

1) Die kurzen Veröffentlichungen über unser Denkmal sind in dem Literaturan: hang am Schluf dieser Studie zusammengestellt. - Zu Tournaires Plan (Bull. 21, pl. XVI/XVII) bemerke ich, daß dort der westlich der Epigonen in situ gezeichnete ca. $2 \mathrm{~m}$ lange antike Rest die willkürlich hingelegten $\delta \varepsilon \approx \dot{\alpha} \tau \alpha \nu$-Quadern bedeuten soll, wogegen unsere wirklich in situ befindliche Mauerecke auf ihnen gänzlich fehlt:

2) Ich habe zunächst auf Grund der Photographie (Abb. 14) an jüngere Zeit geglaubt, aber der Abklatsch zeigt doch ältere Formen, z. B. kein ganz regelmäßiges $N$, sondern mit einem unten etwas schräg auswärts stehendem linkem Schenkel. Diese Eigentümlichkeit stimmt "genau zu den übrigen Tarentiner-Inschriften, von denen selbst die mit $E, A, \oplus$ versehenen schon das gut reglementierte $N$, $\Sigma$ etc. zeigen. Vgl. die Uebersicht in Imagg. ${ }^{3}$ p. 104 f., Nr. 35-38; IGA Nr. 547; Kirchhoff, Stud. ${ }^{4}$ p. 155. Die einzige Ausnahme bilden die Speerspitzen aus der Thurioi-Beute (Olympia $V$ Nr. 254-256), indessen sind auf ihnen die Aufschriften schlecht und flüchtig eingeritzt und ersetzen z. B. alle Rundungen durch Striche $(\circlearrowright=O, \mathbb{D}=\oplus, R=R)$. - S Sodann ist energisch darauf hinzuweisen, daßs die großen, durchlaufenden archaischen $S$ taatsa ufschriften bedeutend sorgfältiger reglementiert zu sein pflegen, als die kleineren Inschriften. Man vergleiche die Inschrift des Stoa der Athener (Beiträge z. Topogr. v. D. Taf. V), deren schönen regelmäfaigen Ductus man früher kaum für möglich gehalten hatte, und dabei wollte man die Stoa in das Jahr 506 setzen. Abgésehen 
die bei den Septem ( 456 v. Chr.) bereits mit der späteren Form ( -1$)$, gemischt auftreten, an unserem Anathem aber, ebenso wie z. B. am platäischen Dreifuß, allein erscheinen. Die Länge der erhaltenen 8 Buchstaben beträgt 1,95 (einschlieklich 0,15 Zwischenraum zum nächsten Zeichen). Das $\Sigma$ vor $\delta \varepsilon x \alpha \dot{\tau} \tau \alpha \nu$ kann man nur auf einen Ortsnamen (z. B. $\ddot{a} \pi \dot{o} \Theta v \varrho \varepsilon ́ \alpha \varsigma, a ̉ \pi \dot{~ O i v o ́ \alpha \varsigma) ~ b e z i e h e n ~ o d e r ~ a u f ~ d a s ~ W o r t ~[v i \varkappa \alpha] \varsigma, ~ b e z w . ~}$ $\left[\mu \alpha^{\prime} \chi \alpha\right]_{S}$ oder auf ein $[\varepsilon \lambda \dot{\delta} \nu \tau \varepsilon] \varsigma$. Außerdem stand der Name der Weihenden voran. Nehmen wir als Beispiel die zeitlich am nächsten stehenden Weiheinschriften der Athener-Halle und des marathonischen Beutesockels, wo in der ersteren das $\tau \dot{\omega} \pi \dot{\jmath} \lambda \lambda \sigma \nu \imath$, in der zweiten das $\dot{\alpha} \nu \varepsilon \dot{v} \varepsilon \sigma \alpha \nu$ fehlte, so erhielten wir als denkbar kürzeste Fassung:

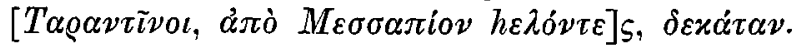

Dabei würden wir für die links fehlenden 29 Zeichen noch 7 Einzelquadern ergänzen müssen, und da auf den 2 erhaltenen Inschriftsteinen je 4 Buchstaben stehen, kommen wir auf eine Inschriftlänge von $9-10 \mathrm{~m}$. Außerdem sind noch mehrere schmalere $(0,82)$ inschriftlose Steine rechts am Ende anzusetzen, von denen Bulle einen auffand. So erhalten wir etwa 12 Platten von $2,40+9$ (bis 10 ) $\mathrm{m}$ Länge, d. h. eine $\mathrm{M}$ in i malfr o $\mathrm{n} t$ von $11-12 \mathrm{~m}$. Da für ein so gewaltig langes Reihenmonument zunächst die 4 Argiveranatheme ausscheiden, - zwei wegen ihrer halbrunden Gestalt, das dritte und vierte (Pferd und Septem) wegen der erhaltenen Weiheinschriften und der Klammerformen -, sodann weder das Lysanderdenkmal - wegen der Klammerform - noch das Marathon-Anathem - wegen des Dialekts - für uns in Betracht kommen kann und von den gut erhaltenen Arkadern ganz zu schweigen ist, so bleiben schlechterdings in diesem ganzen Temenosteil nur die Tarentiner übrig, zu denen die Inschrift gehören könnte. Denn für ein ganz unbekanntes, so riesiges Anathem ist hier nirgends mehr annähernd Platz. Entweder gehören die Quadern zu den Tarentinern, oder sie gehören überhaupt nicht in unsern Temenosteil: tertium non datur. Da letzteres an sich recht unwahrscheinlich ist, und auker den nicht genauer bekannten Liparäer-Anatbemen (Paus. nennt nur ' $\alpha \dot{\nu} \delta \varrho \iota\left(\dot{\nu} \nu \tau \varsigma^{\prime}\right.$ ') kein einziges Weihgeschenk aus dem ganzen Temenos, so weit ich sehe, für unsere lange Standfäche passen würde, da ferner nach den bisherigen Umständen anzunehmen war, daf sich eine oder einige Quadern, vom Tarentinischen Weihgeschenk erhalten haben muliten, und da endlich die vorhandenen Einlabspuren sich für Rosse und Frauen zu eignen schienen, so hielt ich die Zuweisung der Platten zu unserem Denkmal für gesichert.

Leider ging es jedoch auch bei diesem Anathem, wie so oft in Delphi: zuerst schien alles gut zu des Pausanias Beschreibung und den son-

von dem schräg liegenden, aber doch meist mit parall e le n Schenkeln versehenen $\mathrm{N}$, sind die Buchstaben noch schöner reglementiert, als bei unserem $\delta \varepsilon x \alpha \dot{\tau} \tau \alpha v$, und doch ist ihr jü ügst mö gli cher Termin: Salamis, 480 (vgl. A, S, E, l). 
stigen Indizien zu stimmen. Geht man der Sache aber auf den Grund, so kommt gewöhnlich irgend eine technische, archäologische oder historische, epigraphische oder topographische Schwierigkeit zu Tage, die das bisherige Resultat in Frage stellt. So hatte Bulle (ebenso wie Homolle) anfangs geglaubt, die vorhandenen Standspuren mit unserm Anathem in Uebereinstimmung bringen zu können, - später jedoch stellte sich ihm das als unmöglich heraus und er gelangte zu der Ueberzeugung, daf sie vielmehr den eben erwähnten $\mathrm{L}$ i paräern zuzuweisen, also weit verschleppt seien. Ich vermag mich diesem, $\mathrm{n}$ u $\mathrm{x}$ auf der Analyse der Standspuren basierenden Ergebnis noch nicht anzuschlieken, besonders weil die Fundumstände durchaus dagegen sprechen, - und so ist der Ausweg gewählt worden, daß wir, um dem Leser das Material vorzulegen, die Beschreibung dieser Steine am Schluß der Tarentiner belassen unter Anfügung von Bulles Analyse, dak wir aber die endgültige Entscheidung von einer erneuten Prüfung an Ort und Stelle abhängig machen und die $\mathrm{Be}-$ handlung des Liparäer-Denkmals bis zu der ihm zukommenden Studie in Teil II aufschieben. Ueber die Tarentiner läßrt sich, unbeschadet der Frage der Zugehörigkeit jener Quadern, noch folgendes ermitteln:

Z eit, Veranlassung und Künstler der Gruppe. Hier sind wir fast allein auf Pausanias angewiesen, der die zwei Namen Messapier und Ageladas überliefert. Ohne uns auf die früher sehr streitige Chronologie dieses Künstlers genauer einzulassen, dürfen wir soviel als sicher annehmen, daß die von Robert, Busolt, Blümner-Hitzig u. a. vertretene Hinaufdatierung in die Jahre c. $520-480$, gegen den früheren Ansatz c. 500-460, durchaus das Richtige trifft ${ }^{1}$ ). Da auferdem die Tarentiner im J. 473 von den Messapiern die bekannte schwere Niederlage erlitten haben, so gehört unser Anathem zweifellos eine ganze Zeit vor dieselbe, wie ich es bereits Ath. Mitt. 1906, 526, 1 andeutete. Man könnte an die Jahre 500-480 denken, und glauben wollen, dalis Ageladas schon ein berühmter Mann sein mubite, als ihm die Tarentiner den Staatsauftrag erteilten, - aber sein frühestes Werk, von dem wir wissen, hatte auch einem Tarentiner gegolten: es war die Statue des Anochos in Olympia, der 520 im Laufe, später noch einmal im Doppellauf gesiegt hat ${ }^{2}$ ). So stünde nichts entgegen, unser Denkmal nicht nur vor die Schlacht von Marathon, sondern kurz vor den Beginn des 5. Jhdts. zu setzen, wenn

1) Die Literatur ist zusammengestellt von Busolt $\mathrm{II}^{2}, 562,1$, sodann bei BlümnerHitzig, Paus. Bd. II, 1, p. 175 ff.; vgl. auch Robert, Marathonschlacht p. 12. Frazers Erörterungen (Paus. Bd. III $438 \mathrm{ff}$.) sind jetzt überholt.

2) Ueber den blutigen Sieg der Japygier im J. 473 vgl. Herod. VII 170, Diod. XI 52. Ueber die Statue des Tarentiners Anochos Paus. VI 14, 11; Förster, Olympioniken p. 10; Brunn I 63; Robert, Arch. Märchen p. 95 etc. - Ueber die damalige Geschichte von Tarent vgl. Busolt II ${ }^{2}$, 805, der gleichfalls nur unser Anathem vor die grofe Niederlage setzt, während andere auch die 'oberen' Tarentiner vor 473 datieren; vgl. Sauer, Statuar. Gruppe 34, 125. 
nicht der Schrifteharakter der $\delta \varepsilon x a ́ \tau \alpha \nu$-Platte gegen letzteres spräche; diese wird wegen der regelmäfigen Züge (A, E,) kaum in die Nachbarjahre des Korkyrastiers gesetzt werden dürfen, obwohl die Schrift an den verschiedenen Orten verschieden weit reglementiert war. Daber wird man sich mit dem Ansatz in die zweite Lebenshälfte oder in das hohe Alter des Ageladas, d. h. in die z e hn Jahre na ch Marathon (490-480) vorläufig zufrieden geben.

Betreffs der Wahl des A ufstellungs ortes der 'unteren Tarentiner' habe ich die Vermutung, dak sie mit der Nachbarschaft des knidischen Thesauros zusammenhänge. Denn abgesehen von den Thesauren von Sikyon und Siphnos war der ganze Südwestteil des Temenos von Knidos und seinen Kolonien (Lipara) eingenommen. Knidos und Tarent aber waren in jener Epoche auf das engste befreundet, wie die hübsche Geschichte bei Herodot III 138 beweist, wo der verbannte Tarentiner Gillos den Dareios bittet, ihn durch die Knidier in sein Vaterland zurückführen zu lassen, weil deren Fürsprache und Freundschaft daselbst am meisten vermöchten ${ }^{1}$ ). Da wir mehrfach in Delphi die Anatheme befreundeter Staaten als Nachbarn aufgestellt finden, so wäre es wahrscheinlich, dak auch die Tarentiner sich bei ihrem ersten Weihgeschenk die knidische Temenosgegend erwählten, während sie später die Nachbarschaft des Tempels (Plataischer Dreifuf) bevorzugten, weil hier unten alles besetzt war.

Endlich ist zu betonen, das die Darstellung unserer Gruppe von Pausanias nur als 'eherne Pferde und gefangene Frauen' angegeben wird, daßs also alle modernen Variationen abzulehnen sind, die daraus 'Reiter', 'Reiterbilder', 'Krieger' machen wollen. Sie basieren mehr oder minder anf Brunn (I 73), bei dem indessen das Wort Reiter ein einfaches

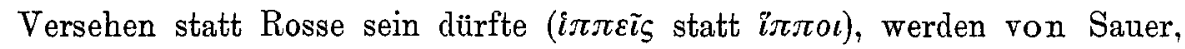
Statuar. Gruppe 33, 124 zusammengestellt und sind zuletzt noch von $\mathrm{Ho}_{0}$

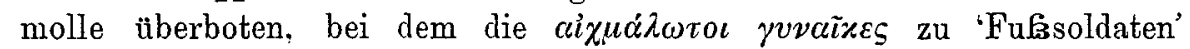
werden (Bull. 22, 579: 'une longue série de cavaliers et de fantassins'). $\mathrm{Zu}$ dem Gegenstand selbst macht $\mathrm{Bull}$ e folgende Bemerkungen:

„Der Sinn des Tarentiner-Weihgeschenks, den man durch A uslegung oder Mißrverständnis des Pausanias zu Unrecht hat verändern wollen, ist sehr einfach: Die Sieger stellen zur Schau, was sie a ls B e ut e von den wilden Messapiern erlangt haben, bei denen es keine Kunst und keine Kostbarkeiten gab, wohl aber köstliche Naturprodukte: Ross e und Weiber. Das bringt einmal eine neue und erfrischende Note in die ewige Heldenwirtschaft der politischen Anatheme und ist so recht im Sinne der immer materiell gesinnten üppigen Grofigriechen. Grade bei diesem ungewöhnlichen Thema hätten wir nun gern einen Anhaltspunkt für die künstlerische Lösung. Da aber die Steine nach meiner Meinung ver-

1) Die Entstehung der Freundschaft führt Busolt $I^{2}, 410,3$ sehr wahrscheinlich darauf zurück, dafs Taras in älterer Zeit vermutlich den Vertrieb knidischer Tonwaren vermittelte. 
sagen, so sind wir auf Vermutungen angewiesen, bei denen wir uns zunächst gegenwärtig halten müssen, dak wir es mit einem a $\mathrm{rch}$ a is ch en Weihgeschenk zu tun haben, also eine gewisse Steifheit und Naivetät der Komposition voraussetzen dürfen. Ferner ergibt die Oertlichkeit, dafi das Anathem eine erhebliche Länge (c. $10-12 \mathrm{~m}$ ) gehabt haben mús.

Rosse und Frauen, als Schaustücke a f f estellt, war also das Thema. Nun könnte man denken, daß die Rosse und die Frauen je für sich eine Gruppe gebildet hätten; das wäre aber selbst für die altertiumliche Kunst wohl zu schwer und eintönig gewesen. Also werden wir annehmen dürfen, dał Rosse und Frauen abwechselten. Die Pferde konnten dann aber kaum mit ihrer Langseite längs der Strafe angeordnet werden, was wiederum höchst sonderbar ausgesehen hätte, sondern sie mußten mit den Köpfen der Straße zugewendet sein. Es ergibt sich dann durch Ausschluß anderer Möglichkeiten etwa folgendes Schema:

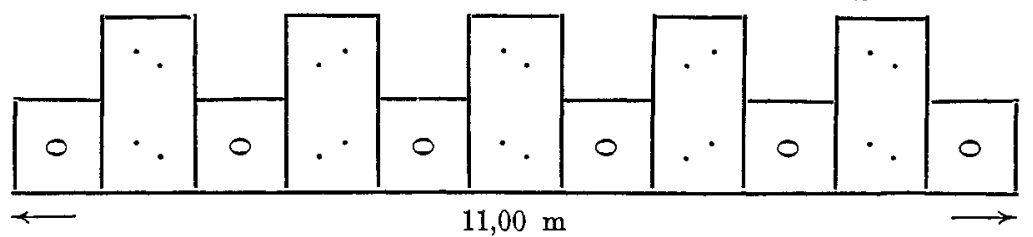

Bei $11 \mathrm{~m}$ Länge hätten wir etwa 5 Rosse und 6 Frauen, indem wir für jede Figur etwa $1 \mathrm{~m}$ Breite ansetzen. Ein Pferd würde die Mitte des Ganzen bilden, an die Ecken kämen Frauen zu stehen. Ieh glaube, daf diese Anordnung eine gewisse innere Wahrscheinlichkeit hat und der feierlichen Grazie des ausgehenden Archaismus recht wohl entspricht. Die Form der Basis mukte sich dabei natürlich dem Gegenstand anpassen, indem die Standflächen der Rosse nach hinten hinausragten. Derartige Anpassung entspricht nicht nur dem Gebrauch der älteren Zeit, sondern läfat sich, soweit mir gegenwärtig, auch durch ein Beispiel wenigstens aus der hellenistischen Epoche belegen (Basis des achäischen Strategen Kallilrates in Olympia, Ergeb. Bd. II, Taf. 94, Nr. 17, Textband II S. 158; Inschr. v. Olymp. Nr. 300). Somit hätten wir uns dies untere Tarentinerweihgeschenk als eine originelle Front von Frauen und Pferdevorderleibern zu denken, in die die Frauen durch Mannigfaltigkeit der Tracht (Peploi und Himatia abwechselnd) einen bewegteren Rhythmus brachten, während die ruhig stehenden Pferde, die wir uns nicht viel höher zu denken haben als die Frauen, mit feierlich steifen Linien dazwischen standen. In diesem Wechsel muks ein nicht geringer Reiz gelegen haben, wobei aber nicht vergessen sei, daß alle diese Aufstellungen nur Vermutungen $\operatorname{sind}^{1}$ ).

Der Aufbau der Basis wird dreistufig gewesen sein, wie wir es bei den andern Anathemen als die Regel kennen. So erhalten wir eine Rekonstruktion des Ganzen, die zwar in allen 'Teilen hypothetisch ist, aber jedenfalls allen gegebenen Daten (Ort, Zeit, Gegenstand und Stil) gerecht wird und unsere Vorstellung von den alten Reihenanathemen immerhin um eine neue Spielart bereichern würde." Bulle.

1) [Ich darf nicht verhehlen, daß mir ein solcher, recht komplizierter, ausgezackter Bathronbau für Delphi und jene alten Zeiten kaum zulässig scheint; er wäre ganz ohne Parallele, denn das Kallikrates-Monument in Olympia stammt aus dem Jahre 179 v. Chr., vermag also für 480 nichts zu beweisen. P.]

K 1i o, Beitráge zur alten Geschichte VIII 3/4. 
Lit er at ur-A nh ang. Nachdem Homollezuerst (Bull.18, 187 vgl. auch Comptes rend. 22, 584) das Monument der Tarentiner auf der rechten (nördlichen) Seite der heiligen Strake angesetzt und die grobe Inschrift $\triangle E K A$. TAN auf dies Anathem bezogen hatte, wies ich nach, daf vielmehr nur die linke Strakenseite in Betracht käme, zwisehen Epigonen und Thesauros von Sikyon (Archäol. Anz. 1895, p. 9). Darauf änderte Homolle seine Ansicht, die inzwischen bei Frazer (Paus. Bd. V p. 269) mißsverständlich dahin erweitert war, daf die Inschrift auf der, rechts die Straße begleitenden, polygonen Stützmauer (Klio VII, 435) gefunden sei und daß das Anathem dort gestanden hätte, - und verwies es auf die von mir bezeichnete Stelle links der Strake (Bull. 21, 301 ff. 〈401〉); zugleich gab er die Inschrift genauer heraus, teilte aber leider keine Fundnotiz mit. Diese Publikation verwertete Frazer in den Nachträgen (Bd. V p. 638). Später kam Homolle nochmals auf unser Monument zu sprechen (Butl. 22, 579) und bezog auf dasselbe jetzt richtig die schon fruher gelegentlich erwähnte polygone Mauerecke; aber aus den Worten "von dem Anathem ist $\mathrm{nur}$ eine Mauerecke übrig, $4,50 \mathrm{~m}$ von der N.W.Ecke des Argiver-Weihgeschenks gelegen; jedoch kann man bezigglich seiner Lage oder Gestalt nicht im Unklaren sein ", scheint hervorzugehen, dalis er die große Inschrift jetzt nicht mehr für z u g e h ö ri g ansieht (?). Ohne dieser Stelle mich zu erinnern, habe ich dann in Delphi sogleich die Mauerecke für zugehörig erklärt (Berl. Phit. Wochenschr. 1906 Sp. 1178, = Delphica p. 27) und die gewaltige Grölie des ganzen Anathems angedeutet.

$\mathrm{D}$ ie Ueberreste der $\delta \varepsilon \varkappa \dot{\alpha} \tau \alpha \nu-\mathrm{S}$ te ine.

(Bisher zu den Tarentinern gehörig.)

Ich schicke einige Mitteilungen aus Kontoleons Inventarabschrift voraus und schliefe daran Bulles Steinbeschreibung und Standspur-Analysen.

In v. Nr. $1629+1425$. - Die zwei Inschriftquadern tragen nicht ein und dieselbe Nummer (1425), wie Homolle angibt, sondern zwei; die dritte, inschriftlose steht nicht im Inventar. - Die zweite Quader Nr. 1425 trägt die Buchstaben ATAN und ist gefunden am 4. Mai a. St. 1894; die Ortsangabe lälist Homolle leider aus, das Inventar aber gibt wieder Rätsel auf. Um dem Leser einen Begriff davon zu geben, welche Mühe oft die kleinsten tatsächlichen Angaben der Studien verursachen, setze ich die griechischen

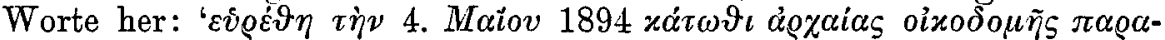

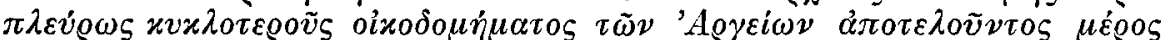

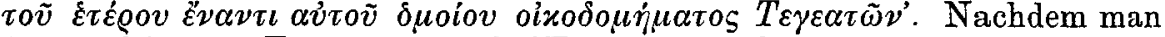
das irreführende $T \varepsilon \gamma \varepsilon \alpha \tau \tilde{\omega} \nu$ durch 'E$\iota \nu$ jó $\nu \omega \nu$ verbessert hat, gelangt man zu dem Fund-Resultat: 'unterhalb der kleinen viereckigen Nische Nr. 7'. Letztere ist Klio VII, $427 \mathrm{ff}$. beschrieben (abgebildet ebenda Taf. I Abb. 14) und liegt genau dem Punkte gegenüber, wo sich die Tarentinerterrasse erhob, bezw. deren Anfang begann. Auch die erste Quader (nr. 1629) mit den Buchstaben $\Sigma \Delta E K$ ist 'im Mai 1894' ebendaselbst gefunden und zwar "nördlich beim Argiverhalbrund".

Dieser Fundort der Platten ist sehr wichtig, denn er spricht a ú erordentlich fürdie Zuweisung zum TarentinerAnathem. Allerdings kommt es bisweilen vor, daß ein größerer Block zur Reparatur der Tempelterrassen-Mauern u. dgl. nach oben transportiert wurde, wie der Stier-Block, der Lysanderstein etc.; auch dati eine einzelne Quader, der 'A 
Studien zu den Weihgeschenken und der Topographie von Delphi. 333

geschleppt wird, ist erklärlich. Darum könnte zufällig auch einmal ei n Stein der Liparaeer von jenseits der Knidos-Siphnos-Thesauren sich hinab zur Königsnische verirrt haben, aber gewiß nicht $\mathrm{zw}$ ei z u sammen$\mathrm{h}$ änge nd e Platten, oder gar eine dritte. Gerade in dieser Gegend, (Tarentiner, Epigonen, Septem, Pferd) war ein Ueberfluis von bequem liegenden, gut behauenen Quadern vorhanden, der es sehr unwahrscheinlich macht, daßi man zur Erbauung der wenigen hier stehenden Dorfhäuser noch Quadern von 'jenseits des Berges' hergeholt hätte. Auch waren unsere Steine nicht verbaut, sondern lagen $\mathbf{u}$ te $\mathrm{rh}$ a $l \mathrm{~b}$ der viereckigen Nische. Ich möchte es für sicher halten, dabi wir, bei einem neuen Besuche, in dieser Gegend noch mehrere solcher Quadern finden werden; und auch die Gegenprobe wird möglich sein, ob sich unter den aufgestapelten Platten unterhalb des Liparaeer-Postaments am Westtor irgendwelche Stuicke nachweisen lassen, die zu unsern Quadern gehört haben können.

Steinbeschreibung und Standspurenanalyse. Von H. Bulle.

Im Jahre 1898 habe ich die drei Steine A, B, C (Abb. 15) als zusammengehörig aufgenommen, von denen A damals dicht am Wege südlich
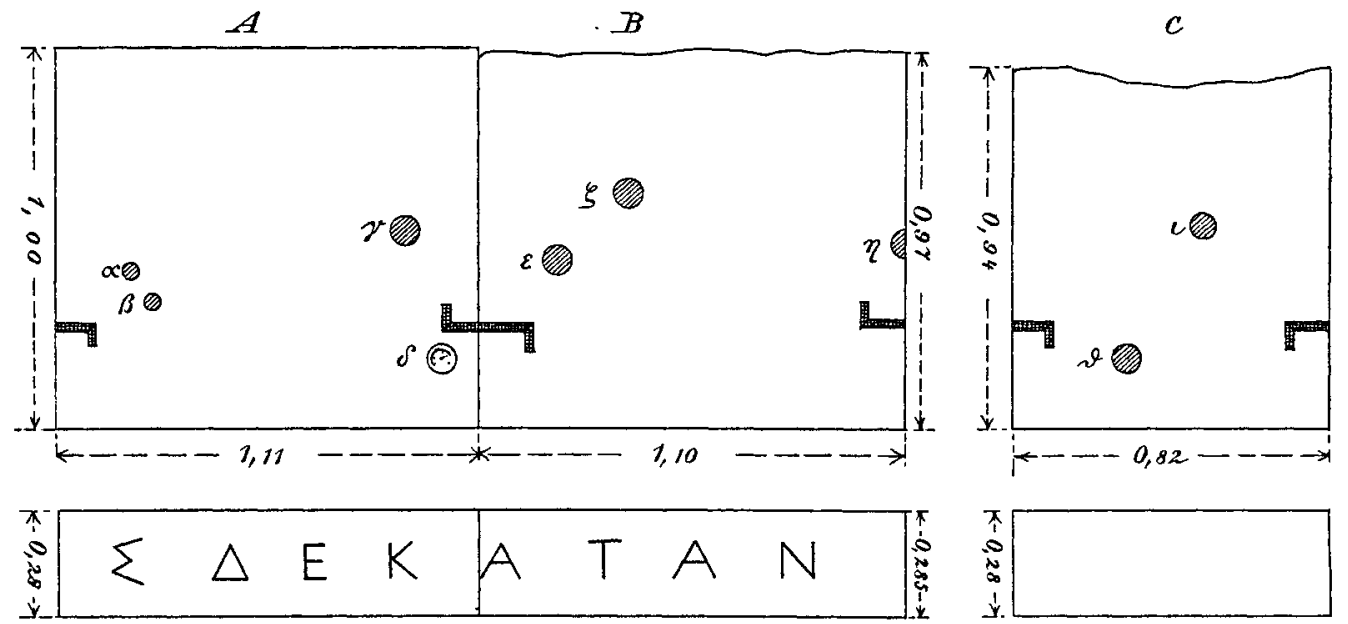

Abb. 15. Die Platten der dqx'́r $\alpha \nu$-Steine. - Maßsstab 1:25.

(Aufnahme von $\mathrm{H}$. Bulle).

der heiligen Stralie, B gegenüber in der viereckigen Nische (Nr. 7), C auf dem Hellenikó etwas westlich von den Epigonen lag. 1903 waren die Steine A und B in der Gegend, wo das grobe Tarentinerweihgeschenk gestanden haben mufi, zusammengelegt. C habe ich 1903 nicht wiedergesehen, allerdings auch nicht danach gesucht.

Die drei Steine A, B, C bestehen aus hartem grauem Kalk mit rötlichen Schichten (Hag. Elias-Stein). Die Höhen sind gleich; sie betragen bei $\mathrm{A}$ und $\mathrm{C} \quad 0,28 \mathrm{~m}$; bei $\mathrm{B}$ habe ich allerdings 0,285 notiert, eine $\mathrm{Ab}$ - 
weichung, die aber entweder auf Ungenauigkeit der Messung, oder auf Kantenverwitterung bei $\mathrm{A}$ und $\mathrm{C}$ zurückgeht, da an der Zusammengehörigkeit wie aus Inschrift, Klammer- und Standspuren hervorgeht, kein Zweifel besteht. Die Breite beträgt bei A $1,11 \mathrm{~m}$, bei B $1,10 \mathrm{~m}$, bei $\mathrm{C} 0,82 \mathrm{~m}$. Die Tiefe scheint nur bei A vollständig erhalten zu sein mit 1,00 m. Die Hinterseite von A ist rauh bearbeitet, jedoch nicht in der Art von Anathyrosis, sondern nur, als ob die Fläche nicht sichtbar gewesen sei. Es hat also nach hinten kein Stein mehr angestoken, was auch daraus hervorgeht, daß hier keine Verklammerung vorhanden ist, wie doch zu den Nachbarsteinen. Jedoch war die Rückseite nicht sichtbar oder nicht zugänglich, wie aus der vernachlässigten Behandlung der Hinterseite hervorzugehen scheint.

Bei $B$ beträgt die Tiefe nur 0,97 und die Rückseite ist ungleichmäßig begrenzt. Da nur $0,03 \mathrm{~m}$ zur vollen Tiefe fehlen und ein so gleichmäßiges Wegbrechen einer dünnen Schicht wenig glaublich ist, so ist hier entweder die Rückseite von Anfang an noch mehr vernachlässigi gewesen oder der schmale Streifen ist etwa bei einer späteren Verbauung abgeschlagen. Sicher abgeschlagen ist die Rückseite von $\mathrm{C}$ mit ihrer unregelmäßigen Grenzlinie, die in der Mitte bis zu $0,12 \mathrm{~m}$ gegen die ursprüngliche Rückseite zurücktritt; die Seitenkanten von $\mathrm{C}$ sind noch 0,94 tief

Auf der Oberfläche sind die 3 Steine jedesmal nach rechts und links durch eine Z-förmige Klammer miteinander verbunden, welche in etwa $0,26 \mathrm{~m}$ Abstand vom vorderen Rande stehen. Und zwar haben die beiden Klammern von A und die linke von B genau diesen Abstand, während ich bei der rechten Klammer von B und der linken von $\mathrm{C}$ den Abstand (mit der geringen Abweichung von $3 \mathrm{~mm}$ ) auf 0,257 m zu messen glaubte, in der Hoffnung, dadurch das Aneinanderpassen von $\mathrm{B}$ und $\mathrm{C}$ erschlieken zu können. Doch ist erstlich, selbst wenn die geringe Differenz trotz der Rauhigkeit des Steins von Ursprung her bestanden hat, dadurch das Anpassen der beiden Steine noch nicht absolut bewiesen, da bei der sehr langen Stufe dieser kleine Unterschied durch Zufall mehrmals auftreten konnte. Zweitens wird das Anpassen sogar unwahrscheinlich, ja unmöglich, weil das Spurloch an der rechten Kante von B nur ein Halbrund ist und sich also auf den Nachbarstein fortsetzen mubte, da alle sonstigen Standlöcher kreisrund sind. Ein halbrundes Spurloch wäre unwahrscheinlich. Die Zusammengehörigkeit von A und B lingegen wird durch die Weihinschrift zur völligen Gewiß̧heit (Buchstabenhöhe 0,10).

Wir haben also die oberste Stufe eines Bathrons vor uns, das eine sehr große Länge gehabt haben muß. Denn zu der Weihinschrift kommt noch mehr hinzu. Zunächst der schriftlose Stein C, den man vor oder nach ihr anordnen kann. Denn da C.kein Eckstein ist, so muf in ersterem Falle, wenn man ihn unmittelbar links vor den Anfang der Weihinschrift stellt, mindestens noch e in weiterer Stein von ungefähr gleicher Breite als Eckstein hinzugefügt werden ${ }^{1}$ ). Ordnet man $\mathrm{C}$ aber am rechten Ende, an, so

1) Pomtow macht darauf aufmerksam, dak die Weihinschrift aber sehr wohl an der linken Ecke beginnen und nur das rechte Ende der Stufe frei lassen konnte. 
sind noch zwei Steine zu ergänzen, da, wie gezeigt, $C$ nicht an $B$ anpakit. Dabi das Postament, auf dem die Steine A-C einst lagen, sehr hoch war, läft sich aus zwei Anzeichen sicher erschließen. Erstens trägt es ganz ungewöhnlicherweise auf der sichtbaren Oberseite Verbindungsklammern, die sonst stets versteckt werden. Bei der Sorgfalt mit der hier sowohl die Standlöcher wie die Inschrift behandelt sind, kann die Abweichung vom gewöhnlichen Gebrauch keine Nachlässigkeit sein, sondern nur den Grund haben, dafs die Stufe ni e m als v on oben betrachtet werden konnte. Zweitens scheint die mangelhafte Bearbeitung der Rückseite darauf hinzuweisen, daf irgend ein Grund den Zugang zu der Hinterseite des Bathron verhinderte.

$\mathrm{Di}$ e $\mathrm{Standspuren}$ bestehen aus kreisrunden Löchern von 0,065 bis $0,085 \mathrm{~m} \mathrm{Dm}$. und $0,10-0,11 \mathrm{~m}$ Tiefe $(\gamma=$ Dm. 0,$07 ; \mathrm{t} .0,11 . \delta=$ Dm. 0,08; Rest eines halbrunden Bronzezapfens, sonst mit Blei gefüllt. $\varepsilon=$ Dm. 0,07 ; t. $0,105 . \quad \zeta=$ Dm. 0,085 ; mit Blei gefüllt. $\eta=$ Dm. 0,075 ; t. 0,115 ; nur zur Hälfte erhalten. $\vartheta=$ Dm. 0,075 ; t. $0,10 .:=$ Dm. 0,065; t. 0,10 ). Kleiner sind die beiden Löcher $\alpha$ und $\beta$ (je $0,045 \mathrm{Dm}$., 0,045 T.). Aus ibrer Verteilung sieht man sofort, dak es sich hier nicht um das gewöhnliche Aneinanderreihen von stehenden Männern handeln kann, denn höchstens $\varepsilon-\zeta$ ergäben eine normale Standfigur. Aber da diese Löcher so eng mit $\gamma-\delta$ gruppiert sind, so muls mindestens eine enge Beziehung zwischen $\varepsilon-\zeta$ and $\gamma-\delta$ bestehen. Man beginnt die Betrachtung am besten mit $\vartheta-\iota$ anf $C$. Die Löcher stehen etwa $40 \mathrm{~cm}$ auseinander. $\vartheta$ ist etwas größer, $\iota$ etwas kleiner. Für ein Pferd oder sonstiges Tier wükte ich die Spuren nicht zu erklären, da sie zu weit auseinanderstehen. Für ein gewöhnliches statuarisches Motiv ist die Spreizung zu grof. Es bleibt also eine schreitende menschliche Gestalt, deren Hauptlast von dem dickeren vorderen Zapfen $\vartheta$ getragen wurde. Es muls also etwa ein Krieger oder Held in Kampfstellung gewesen sein. Auffallend wäre höchstens, daß3 die Bewegung fast im rechten Winkel zur Basis, nach vorne und auken, geht. Am wahrscheinlichsten scheint mir daher folgendes: der Krieger wich von einem von rechts kommenden Gegner zurück, sein rechter Fufs stand in $\vartheta$, das rechte Knie war eingeknickt und trug die Hauptlast des sich zurückbiegenden Oberkörpers; der linke Fuf stand mit gestrecktem Bein auf $\iota$. Das ergibt ein sehr geläufiges Kampfmotiv, wie es in den großen Friesen hänfig ist, ein Zurückbiegen aus der Ausfallstellung vor einem stark andringenden Feind. Natürlich hat diese Erklärung nicht den Wert völli ge r Sicherheit, da uns hier ja leider die sonst so sprechenden Fußumrisse fehlen. Aber da der Kreis der Dinge, die überhaupt dargestellt sein $\mathrm{k} \ddot{0} \mathrm{n} \mathrm{n} \in \mathrm{n}$, ja ein ganz beschränkter ist Tiere, stehende Männer, kämpfende Männer, Frauen, - so hat die gegebene Deutung doch die allergrößte Wahrscheinlichkeit für sich.

Ebenso verhält es sich mit $\gamma-\delta-\varepsilon-\zeta$. Ein Tier ist auch hier ausgeschlossen. Nimmt man ruhigstehende Figuren an, so könnte man ja allerdings in $\varepsilon-\zeta$ eine Gestalt mit annähernd normaler Haltung anbringen. Aber wie sollte sich $\gamma-\delta$ dazu verhalten? Soll diese Statue der ersten die Brust oder den Rücken zukehren, während sie selbst weit ausschreitet 
$(0,36 \mathrm{~m})$ ? Auch hier kommt man nur zu einer glaubhaften Annahme, wenn man eine Ka $\mathrm{mpfgruppe}$ anzubringen versucht. Entweder trugen $\gamma-\delta$ und $\varepsilon-\zeta$ je einen Gegner, die sich in Ausfallstellung nah auf den Leib gerückt und buchstäblich handgemein geworden sind. Oder man teilt die beiden hinteren Löcher $\gamma-\zeta$ dem einen, die vorderen $\delta-\varepsilon$ dem andern zu. Der hintere Krieger müsste dann weit ausschreitend (von rechts nach links) einen schon besiegten und zusammenbrechenden Feind unter sich haben; auch dies ist eine Gruppe, für die jedem sogleich $\nabla$ orbilder einfallen. Gewiß lassen sich auch noch andere Möglichkeiten aussinnen, sie müssten sich aber alle in diesem Kreise eines kämpfenden Paares bewegen. - Das halbe Loch $\eta$ auf B liegt zu weit von den vorigen Iü̈chern ab, um mit ihnen zusammenzuhängen und kann in seiner Vereinzelung nichts lehren.

Sehr lehrreich dagegen sind die beiden kleinen Löcher $a-\beta$. Mit der vermuteten Kampfgruppe $\gamma-\zeta$ können sie gar nichts zu tun haben, sondern stehen soweit von allen übrigen ab, dab etwas Selb ständ i g e s darauf gewesen sein muk. Ein menschlicher Fub kann dies keinesfalls gewesen sein. Denn selbst wenn man annehmen wollte, dafs hier ausnahmsweise zwei statt eines Zapfens verwendet wären, so hätten diese Zapfen doch zu geringe Tiefe $(0,045)$, um eine Statue von der Gröke der übrigen (Zapfentiefe 0,10 bis 0,11 ) zu halten. Somit bleibt für $\alpha-\beta$ nur ein lebloser Gegenstand übrig, der schräg zu der Lüngsachse der Basis nach vorne ragte. Aus den Spurlöchern kann er nicht erraten werden, vielmehr müssen wir jetzt unter den überlieferten Anathemen Umschau halten, bei denen jedoch die Tarentiner ausscheiden, da Rosse a uf diesen Spuren ganz unmöglich, Frauen völlig unwahrscheinlich sind. Es bleibt im ganzen unteren Teil des heiligen Bezirks nur noch ein einziges großes Anathem übrig, nämlich das der L i p a rä er, von dem Paus. X

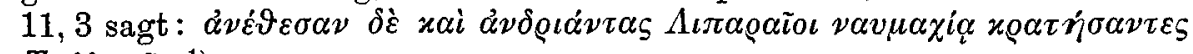

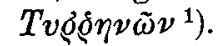

Da Pausanias nur von $\ddot{a} \nu \delta \varrho \iota \dot{\nu} \nu \varepsilon \varsigma$ spricht und keine Heroennamen oder dgl. nennt, können es keine mythischen Repräsentanten gewesen sein, deren bloße Erscheinung schon genügend gesprochen hätte, sondern es müssen Vertreter der Schlacht selbst, d. h. die Liparäer in Person gewesen sein. Da es aber nicht angeht, daß einzelne ruhigstehende menschliche Krieger ein volles Symbol des Ereignisses sind, so kann eben nur die Schlacht selbst dargestellt gewesen sein. Eine Seeschlacht als solche ist aber wiederum in der Plastik nicht denkbar. Also bleibt nur übrig, daf es einzelne $\mathrm{Kampfgruppen}$ gewesen sind, wie sie ja auch in der Wirklichkeit beim Entern der Schiffe vorkamen. Die Andeutung der Seeschlacht geschah, wie so oft auf Vasenbildern, zweifellos durch Anbringung von einzelnen Schiffsteilen, Schnäbeln und Rammspornen oder den malerisch aufgebogenen Heckverzierungen ( $\ddot{x} \varphi \lambda \alpha \sigma \tau o \nu$, aplustre).

1) Aus einer Veranlassung ganz gleicher Art hatten die Liparäer ein zweites Weibgeschenk oben auf der großen Tempelterasse gestiftet, 20 Apollonstatuen, gleich der Anzahl der damals besiegten und genommenen Schiffe der Tyrrener, die sich in sonderbarem Stolze, pentadenweise von den Liparäern hatten abtun lassen (X 16, 7). 
Studien zu den Weihgeschenken und der Topographie von Delphi. 337

Verglichen mit diesem Lipara-Anathem fänden nun die Standspuren ihre Erklärung: ein Einzelkämpfer auf C, dessen Gegner fehlt, ein kämpfendes Paar auf $\mathrm{A}-\mathrm{B}$ und endlich in $\alpha-\beta$ auf $\mathrm{A}$ ein lebloser Gegenstand von selbständiger Bedeutung, in dem wir nun ein Aplustre oder sonstigen Schiffsteil annehmen werden. Es müßte doch ein sonderbarer Zufall sein, wenn diese Uebereinstimmungen nicht der Wirklichkeit entsprächen. Die ganze übrige Reihe des Anathems ist dann eben so zu ergänzen: $\mathrm{k} a ̈ m p f$ e $\mathbf{n d e}$ Gruppen, unterbrochen von einzelnen Teilen von Schiffen, durch die das Ganze als eine Seeschlacht deutlich wird.

Daki die verstümmelte Weihinschrift sich auch für das lipara-Anathem ergänzen lälst, liegt auf der Hand. Aus der nicht ganz gewöhnlichen

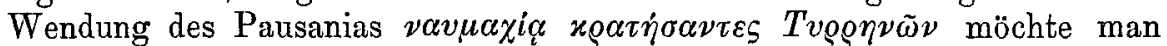
auf folgenden Wortlaut der Weihung schliefen:

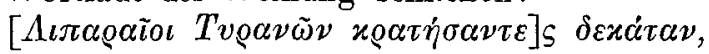

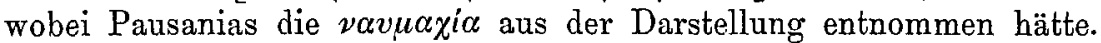

Jedoch mufi die Fortführung des Wahrscheinlichkeitsbeweises hier abgebrochen werden. Denn da Pomtow und Wolters eine lange Basis am Westtor (südlich des Weges) vermutungsweise den Liparäern zuweisen (oben p. 81,2), diese Reste also in den II. Teil des Temenos gehören, so erfolgt der Abdruck der weiteren Untersuchung über die Zugehörigkeit unserer Steine, sowie über Zeit und Veranlassung des Lipara-Anathems besser erst bei der Behandlung jenes Postaments. Bulle.

Das Weihgeschenk der Tarentiner ist das letzte in dem ersten Teil des Temenos, der bis zum Thesauros von Sikyon reicht, gewesen, soweit die groken, in situ befindlichen und z. T. von Pausanias erwähnten Anathemata in Frage kommen. Das Sikyon-Schatzhaus selbst wird passend einen neuen Teil der Studien eröffnen, der hauptsächlich architektonischer Natur sein und die meisten Thesauren (Sikyon, Siphnos, Knidos, Boeotien, Athen, Syrakus, Potidaea, Caere-Agylla) enthalten wird.

Zunächst werden einige kleinere Weihgeschenke (Nr. 18-25), die zwischen und neben den groken Kammern und Unterbauten des ersten TemenosTeils aufgefunden oder zu ergänzen sind, als Schlußabschnitt zum Vorstehenden angefügt ${ }^{1}$ ), können aber nur kurz besprochen werden.

1) Klio IX Heft 1. 